\title{
Analytical Characterization of ITLinQ: \\ Channel Allocation for Device-to-Device Communication Networks
}

\author{
Ratheesh K. Mungara, Student Member, IEEE, Xinchen Zhang, Member, IEEE, \\ Angel Lozano, Fellow, IEEE, and Robert W. Heath, Jr., Fellow, IEEE
}

\begin{abstract}
Device-to-device (D2D) communication allows serving local wireless traffic bypassing the system's infrastructure. One way to control the interference in D2D networks is to carefully channelize transmissions. This paper presents an analytical characterization of ITLinQ, one of the principal D2D channelization schemes proposed to date. Recognizing that it captures well the spatial characteristics of D2D networks, a stochastic geometry setting is utilized for this analysis. The derived expressions enable gleaning insights on how ITLinQ avoids situations of excessive interference, and they facilitate optimizing the controllable parameters of ITLinQ so as to maximize the system spectral efficiency (bits/s/Hz per unit area). With the parameters thus optimized, the ultimate performance of ITLinQ can be evaluated with respect to other D2D channel allocation schemes. In particular, performance evaluation comparisons with the FlashLinQ scheme are provided, and the gains with respect to an unchannelized network are quantified.
\end{abstract}

\section{Index Terms}

R. K. Mungara and A. Lozano are with the Department of Information and Communication Technologies, Universitat Pompeu Fabra (UPF), 08018 Barcelona, Spain. E-mails: \{ratheesh.mungara, angel.lozano\}@upf.edu.

X. Zhang was with The University of Texas at Austin. He is now with Qualcomm Inc., San Diego, CA 92121, USA. Email: xzhang7@alumni.nd.edu.

Robert W. Heath Jr. is with The University of Texas at Austin, Austin, TX 78704-0240. Email: rheath@ece.utexas.edu.

This work was supported in part by Intel's University Research Program "5G: Transforming the Wireless User Experience", by the MINECO Project TEC2012-34642 and by the National Science Foundation Grant No. 1218338.

Parts of this paper were presented at the 2014 Asilomar Conference on Signals, Systems and Computers and at the 2015 International Conference on Communications (ICC'15). 
ITLinQ, FlashLinQ, channelization, channel allocation, stochastic geometry, Poisson point process, spectral efficiency.

\section{INTRODUCTION}

The incorporation of D2D (device-to-device) communication into infrastructure-based wireless networks promises major performance advantages provided there is sufficient locality in the traffic [1]-[7]. Both academia and industry have conducted studies on D2D to support applications such as multicasting [8], [9], content distribution [10], [11], cellular offloading [12], machine-to-machine communication [13], [14] or proximity-aware internetworking [5], [15], [16]. While much of this prior work concentrated on underlay communication where D2D transmissions reuse the existing uplink spectrum [2], [17], [18], there is growing interest in overlay alternatives [3], [19]. In overlay mode, a swath of spectrum is reserved for D2D traffic, which is thereby segregated from both uplink and downlink. On this dedicated spectrum, users in close proximity can establish direct communication, replacing two long hops via the infrastructure with a single (and typically shorter) hop. Such direct communication can improve the power efficiency [20], the end-to-end latency [12], and especially the spectral efficiency, all thanks to the reduced range and denser spectral reuse [21]. However, without a careful allocation of D2D links to time/frequency signaling channels, a process that we shorten as channelization, a significant share of the D2D links may experience strong interference from other unintended D2D transmissions that happen to originate nearby. And, as the density increases, this interference could progressively clog the network.

Channelization schemes have been studied in the context of wireless ad-hoc networks, where fixed infrastructure is absent [22]-[27], and cognitive networks [28], [29]. This prior work had focused either on improving transmission capacity [22]-[25], [28], [29], defined as the maximum permissible density of simultaneous transmissions that satisfies a target receiver SIR (signal-to-interference ratio) with a specified outage probability, or else on analyzing interference statistics [26]-[28]. With the growing interest in overlaid D2D, new schemes are being discussed that can befit networks with an infrastructure-supported control plane and the ensuing ability to synchronize transmissions, discover neighbors, and disseminate side information.

A first such scheme, termed FlashLinQ, was formulated in [30], [31] and experimentally demonstrated. A subsequent scheme, referred to as ITLinQ, was proposed in [32] and evaluated by means of Monte-Carlo simulations. This latter scheme is enticing because it is underpinned 
by information-theoretic optimality notions, which potentially makes it $(i)$ more suitable to analysis, with the ensuing broader generality and with the posssibility of optimizing controllable parameters, and (ii) more apt to provide insight and understanding on the mechanisms exercised to manage interference. These arguments motivate the analysis of ITLinQ that we present here, with the following contributions:

- We derive the exact density of D2D links allowed under different ITLinQ implementations for both fixed and randomized intended link distances. In most previous analyses of channelization in ad hoc networks [24], [27], [28], every link had the same distance.

- By means of the link density expressions, the spatially averaged link spectral efficiency (b/s/Hz per link) and system spectral efficiency (b/s/Hz per unit area) are approximated in integral forms. Numerical results confirm the accuracy of these approximations.

- Based on the system spectral efficiency expressions, the ITLinQ parameters are tuned as function of the user density and link distance distribution.

- With the parameters optimized, the performance of ITLinQ is evaluated and compared with that of FlashLinQ, which we also optimize for this comparison. Both ITLinQ and FlashLinQ are shown to yield substantial improvements with respect to an unchannelized baseline, with a slight edge for ITLinQ over FlasLinQ.

Above all, the analysis in this paper (prequels of which were presented in [33] and [34]) sheds light on the mechanisms that ITLinQ utilizes to shield users from excessive interference while attempting to pack as many concurrent D2D transmissions per unit area as possible.

\section{SYSTEM MODEL}

Recognizing that stochastic geometry allows for models that are both amenable to analytical treatment and highly representative of the spatial behavior of D2D users, this is the toolbox that we invoke for our analysis.

\section{A. User Spatial Distribution}

We consider the Poisson bipolar model [35] where D2D transmitters are spatially distributed according to a marked homogeneous PPP, where "marked" means that each point is labeled with a mark whose distribution features additional information such as transmit power, fading, or link priority [36]. We define the process of D2D transmitters as $\hat{\Phi}=\left\{\left(x_{k}, \theta_{k}, m_{k}, e_{k}\right)\right\} \subset$ $\mathbb{R}^{2} \times[0,2 \pi) \times[0,1] \times\{0,1\}$ where: 
- $\Phi=\left\{x_{k}\right\} \subset \mathbb{R}^{2}$ is the uniform PPP with intensity $\lambda$ representing the locations of all existing transmitters with $x_{k}$ the location of the $k$ th-link transmitter.

- $\theta_{k}$ denotes the IID (independent identically distributed) orientation angle, uniformly distributed in $[0,2 \pi)$, between the transmitter at $x_{k}$ and its intended receiver.

- $m_{k}$ is an IID random mark associated with the $k$ th link, uniformly distributed in $[0,1]$, which may represent the time stamp or the priority of the $k$ th link.

- $e_{k} \in\{0,1\}$ is a retaining indicator that indicates whether the $k$ th link is allowed on a given channel.

Denoting by $r_{v_{k}, x_{k}}$ the distance between the $k$ th-link transmitter and its intended receiver, the location of such receiver is $v_{k}=x_{k}+\left[r_{v_{k}, x_{k}} \cos \theta_{k}, r_{v_{k}, x_{k}} \sin \theta_{k}\right]^{T}$. In the absence of empirical data on how $r_{v_{k}, x_{k}}$ is distributed, various canonical distributions have been entertained in the literature: Rayleigh distributions [37]-[39], inverse functions of the link density [21], [32], or uniform distributions within a circle centered on the transmitter [40]. In this paper, we consider the flexible discrete marginal distribution (IID across links)

$$
r_{v_{k}, x_{k}}=d_{n} \quad \text { with probability } \quad p_{n}
$$

and with $\sum_{n=1}^{N} p_{n}=1$. By choosing $N$ and the appropriate values for $d_{1}, \ldots, d_{N}$ and $p_{1}, \ldots, p_{N}$, we can reproduce as approximately as desired the behavior of any of the aforementioned continuous distributions. We hasten to emphasize that each link's distance is independent of the rest, with their distributions IID.

\section{B. Signal Model}

By Slivnyak's Theorem [36], we can place at the origin a receiver and center the analysis on its link, indexed by 0 . This link, whose transmitter is located at $x_{0}$, can serve as the typical link in the network.

Denoting by $P$ the signal power measured at $1 \mathrm{~m}$ from its transmitter, the receiver at the origin observes

$$
y_{0}=\sqrt{P r_{v_{0}, x_{0}}^{-\eta}} h_{v_{0}, x_{0}} s_{0}+z_{0}^{\prime}
$$

whose first term is the intended signal while the second term is the aggregate interference plus noise

$$
z_{0}^{\prime}=\sum_{k=1}^{\infty} \sqrt{P r_{v_{0}, x_{k}}^{-\eta}} e_{k} h_{v_{0}, x_{k}} s_{k}+z_{0}
$$


where the summation spans the co-channel transmitters in $\Phi \backslash\left\{x_{0}\right\}$ while $z_{0}$ denotes the AWGN. In turn, $\eta>2$ is the pathloss exponent, $r_{v_{0}, x_{k}}$ is the distance between $v_{0}$ and $x_{k}$ (i.e., from the transmitter at $x_{k}$ to the receiver at $\left.v_{0}\right), h_{v_{0}, x_{k}}$ denotes the corresponding fading, and $s_{k}$ is the data symbol communicated over the $k$ th link. The fading coefficients are IID complex Gaussian with zero mean and unit variance, i.e., $h_{v_{0}, x_{k}} \sim \mathcal{N}_{\mathbb{C}}(0,1)$. Likewise, $s_{k} \sim \mathcal{N}_{\mathbb{C}}(0,1)$ and $z_{0} \sim \mathcal{N}_{\mathbb{C}}\left(0, F_{\mathrm{N}} N_{0} B\right)$ where $F_{\mathrm{N}}$ is the noise figure, $N_{0}$ is the noise power spectral density and $B$ is the bandwidth.

The local-average SNR (signal-to-noise ratio) at the 0th-link receiver is

$$
\mathrm{SNR}=\frac{P}{F_{\mathrm{N}} N_{0} B} r_{v_{0}, x_{0}}^{-\eta}
$$

Shadow fading, not considered here, could possibly be incorporated by adopting the approach in [41]. Since it can be seen as a distortion of the spatial geometry, shadow fading would render any circular region amorphous and modify the quantitative results, yet we would expect it not to alter the intuition and the qualitative assessments. This is indeed what has been observed in other stochastic geometry analyses, e.g., in [42].

\section{ITLINQ ANALYSIS}

\section{A. Description}

ITLinQ is grounded on a certain information-theoretic optimality notion: it selects, for allocation to each channel, a subset of links whose mutual interference can be treated as noise while still achieving the capacity region of that subset to within a constant gap [32]. That amounts to assigning the 0th link to a channel if and only if its transmitter and receiver satisfy the necessary and sufficient condition

$$
\mathrm{SNR} \geq \max _{k \neq 0} \mathrm{INR}_{0, k}^{\text {in }} \max _{i \neq 0} \mathrm{INR}_{i, 0}^{\text {out }}
$$

where $I N R_{0, k}^{\text {in }}$ is the incoming local-average INR (interference-to-noise ratio) from link $k$ to link 0 , i.e., the INR from the $k$ th-link transmitter to the 0th-link receiver, given by

$$
\mathrm{INR}_{0, k}^{\text {in }}=\frac{P}{F_{\mathrm{N}} N_{0} B} r_{v_{0}, x_{k}}^{-\eta}
$$

while $\mathrm{INR}_{i, 0}^{\text {out }}$ is the outgoing local-average INR to link $i$ from link 0 , i.e., the INR from the 0th-link transmitter to the $i$ th-link receiver

$$
\mathrm{INR}_{i, 0}^{\text {out }}=\frac{P}{F_{\mathrm{N}} N_{0} B} r_{v_{i}, x_{0}}^{-\eta}
$$


To implement (5), a centralized controller having local-average channel knowledge of all the links would be required. To relax this requirement, a distributed version of ITLinQ was proposed in [32, Sec. III] where each transceiver can check its own condition locally with respect to already retained links, in a sequential fashion. This distributed version of ITLinQ relies only on two sufficient conditions, derived from (5), which expressed for the 0th link amount to

$$
\begin{aligned}
& \mathrm{SNR}^{1 / 2} \geq \max _{k \neq 0} \mathrm{INR}_{0, k}^{\text {in }} \\
& \mathrm{SNR}^{1 / 2} \geq \max _{i \neq 0} \mathrm{INR}_{i, 0}^{\text {out }}
\end{aligned}
$$

where (8) must be satisfied at the receiver of link 0 while (9) must be satisfied at the corresponding transmitter. Because distributed ITLinQ is based on sufficient but not necessary conditions, it is overly conservative and hence it experiences a certain loss relative to centralized ITLinQ. This loss can be eliminated almost completely by heuristically modifying the sufficient conditions in (8) and (9) into [32]

$$
\begin{array}{ll}
M \mathrm{SNR}^{\mu} \geq \mathrm{INR}_{0, k}^{\text {in }} & \forall k \neq 0 \\
\operatorname{MSNR}^{\mu} \geq \mathrm{INR}_{i, 0}^{\text {out }} & \forall i \neq 0
\end{array}
$$

where $M$ and $\mu$ are positive parameters.

Two slightly different distributed forms of ITLinQ are considered here, inspired by the Matérn hard-core processes of type I and type II that are widely utilized to analyze CSMA [26], [27].

- ITLinQ type I, whereby the conditions are applied to all existing links simultaneously rather than in a sequential fashion. The 0th link is allowed in a channel if and only if conditions (10) and (11) are satisfied with respect to all existing transmitters or receivers. If not allowed in a given channel, then the 0th link is served on another channel and thus we can regard the analysis herein as corresponding to the channel where this link is served. On such channel, the network that results with type I channelization is indicated by $\hat{\Psi}_{\mathrm{I}}=\left\{\left(x_{k}, \theta_{k}\right):\left(x_{k}, \theta_{k}, e_{k}\right) \in \hat{\Phi}\right.$ and $\left.e_{k}=1\right\}$ while the process of co-channel transmitter locations is represented by $\Psi_{\mathrm{I}}=\left\{x_{k}:\left(x_{k}, \theta_{k}\right) \in \hat{\Psi}_{\mathrm{I}}\right\}$.

- ITLinQ type II, whereby the sequentiality in the application of the conditions is replaced by a prioritization embodied by the marks $m_{k}$. Rather than against all links, the conditions are verified against lower-priority links, i.e., the links are prioritized by the network and the Oth link is allowed in a channel if and only if conditions (10) and (11) are satisfied with respect to the subset of transmitters or receivers with lower mark, $i, k \in\left\{n: m_{n}<m_{0}\right\}$. 
On the channel serving the 0th link, the network that results with type II channelization is indicated by $\hat{\Psi}_{\mathrm{II}}=\left\{\left(x_{k}, \theta_{k}\right):\left(x_{k}, \theta_{k}, m_{k}, e_{k}\right) \in \hat{\Phi}\right.$ and $\left.e_{k}=1\right\}$ while the process of co-channel transmitter locations is represented by $\Psi_{\mathrm{II}}=\left\{x_{k}:\left(x_{k}, \theta_{k}\right) \in \hat{\Psi}_{\mathrm{II}}\right\}$.

\section{B. Geometric Interpretation}

Plugging into the condition in (10) the definitions of SNR and INR $\mathrm{R}_{0, k}^{\mathrm{n}}$ given in (4) and (6), respectively, we obtain

$$
r_{v_{0} x_{k}} \geq \frac{r_{v_{0} x_{0}}^{\mu}}{M^{1 / \eta}}\left(\frac{P}{N_{0} F_{\mathrm{N}} B}\right)^{\frac{1-\mu}{\eta}}
$$

leading to the equivalent condition

$$
r_{v_{0} x_{k}} \geq \mathrm{r}
$$

with

$$
r=\frac{r_{v_{0}, x_{0}}^{\mu}}{M^{1 / \eta}}\left(\frac{P}{N_{0} F_{\mathrm{N}} B}\right)^{\frac{1-\mu}{\eta}}
$$

Thus, ITLinQ surrounds the receivers with exclusion regions of radius $r$ where either no transmitter whatsoever is present (type I) or no lower-priority transmitters are present (type II). Likewise, by plugging (4) and (7) into (11), it can be verified that ITLinQ surrounds the transmitters with exclusion regions of radius $r$ where either no receiver is present (type I) or no lower-priority receivers are present (type II).

These exclusion regions, illustrated in Fig. 1, curb interference from all other links (type I) or from lower-priority links (type II) and ensure that conditions (10) and (11) are satisfied as far as those links are concerned. As seen in Fig. 1, type II is less conservative than type I and allows for a tighter packing of co-channel links by means of link prioritization.

\section{Co-Channel Link Density}

The first and pivotal result we present characterizes the density of links that end up coexisting on a given channel when ITLinQ is applied. Let $\mathcal{B}_{x}(\mathrm{r})$ denote a circle centered at $x$ with radius $r$ and let $\mathbb{1}(\cdot)$ be the indicator function returning 1 if its argument is true and 0 otherwise. 


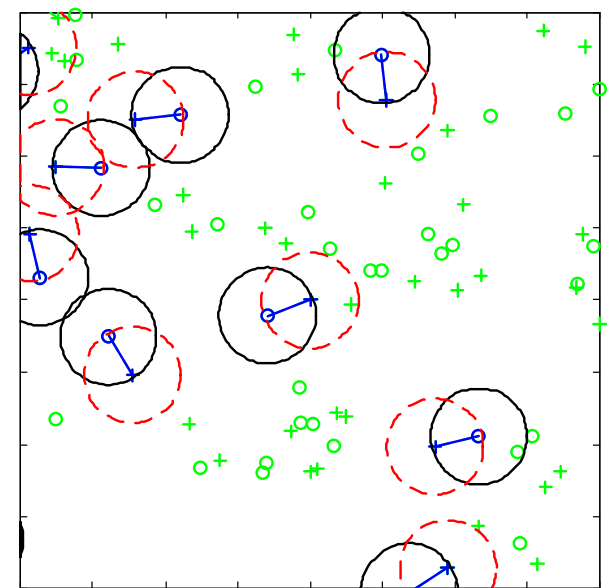

(a) Type I

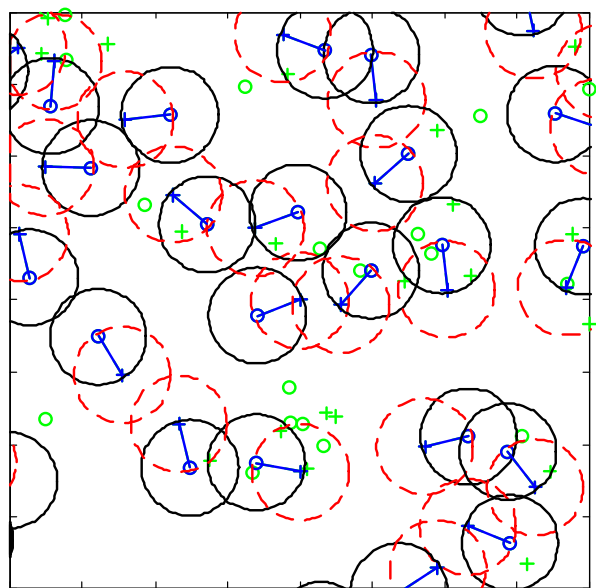

(b) Type II

Fig. 1: Channelized network realization under ITLinQ. Transmitters and receivers are indicated by $\circ$ and + , respectively. Links allowed to coexist on the channel of interest are connected by solid lines while other links are not connected. Solid and dashed circles represent the exclusion regions around transmitters and receivers, respectively. In this example, and only for illustrative purposes, every link has the same distance and hence all exclusion regions are equally sized.

Lemma 1. If the link distance is distributed according to (1), then the co-channel link densities of ITLinQ types I and II equal

$$
\begin{aligned}
\lambda_{\Psi_{\mathrm{I}}} & =\sum_{n=1}^{N} p_{n} \lambda e^{-\lambda A\left(\mathrm{r}_{n}, d_{\ell}\right)} \\
\lambda_{\Psi_{\mathrm{II}}} & =\sum_{n=1}^{N} \frac{p_{n}}{A\left(\mathrm{r}_{n}, d_{\ell}\right)}\left(1-e^{-\lambda A\left(\mathrm{r}_{n}, d_{\ell}\right)}\right)
\end{aligned}
$$

where $r_{n}$ is the exclusion region radius corresponding to $r_{v_{0}, x_{0}}=d_{n}$ in (14) while

$$
A\left(\mathbf{r}_{n}, d_{\ell}\right)=\pi r_{n}^{2}+\sum_{\ell=1}^{N} p_{\ell} \int\left(1-\mathbb{P}\left(v_{i} \notin \mathcal{B}_{x_{0}}\left(r_{n}\right) \mid x_{i}, r_{v_{i}, x_{i}}=d_{\ell}\right)\right) \mathrm{d} x_{i}
$$

with integration over $\mathcal{B}_{x_{0}}\left(\mathrm{r}_{n}+d_{\ell}\right) \backslash \mathcal{B}_{v_{0}}\left(\mathrm{r}_{n}\right)$ and with

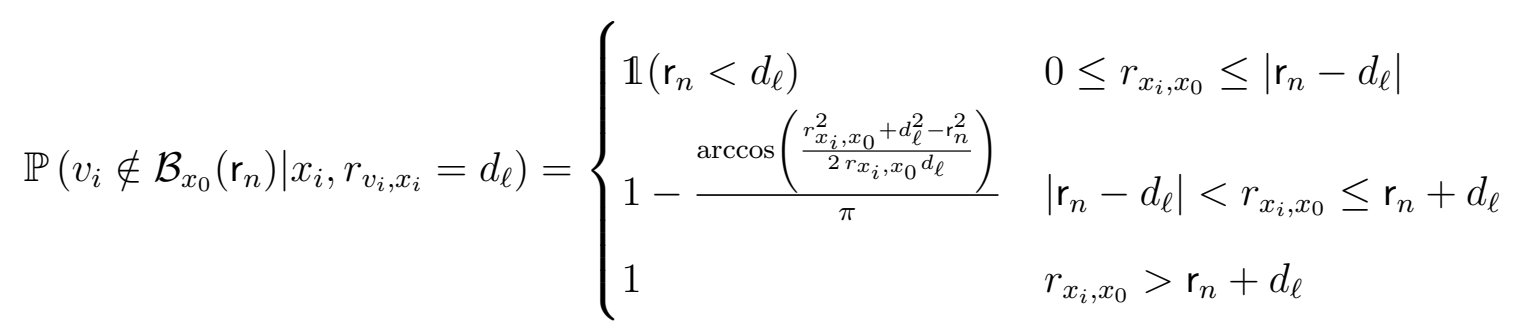


the probability that the receiver of the ith link is not in $\mathcal{B}_{x_{0}}\left(r_{n}\right)$, conditioned on the location of the ith-link transmitter $x_{i}$.

Proof. See Appendix A.

TABLE I: Co-Channel Link Density (links $/ \mathrm{km}^{2}$ )

\begin{tabular}{|c|c|c|c|c|}
\hline & \multicolumn{2}{|c|}{ Fixed link distance } & \multicolumn{2}{c|}{ Random link distance } \\
\hline$(M, \mu)$ & $\lambda_{\Psi_{\mathrm{I}}}$ & $\lambda_{\Psi_{\mathrm{II}}}$ & $\lambda_{\Psi_{\mathrm{I}}}$ & $\lambda_{\Psi_{\mathrm{II}}}$ \\
\hline \hline$(10,0.5)$ & 8.61 & 10.00 & 8.69 & 10.04 \\
\hline$(10,0.7)$ & 10.09 & 10.80 & 10.13 & 10.82 \\
\hline$(10,0.9)$ & 10.88 & 11.21 & 10.86 & 11.20 \\
\hline
\end{tabular}

Example 1. Let $\lambda=11.54$ links $/ \mathrm{km}^{2}$, which amounts to an average of 10 D2D users per circular cell of radius $525 \mathrm{~m}$, with a pathloss exponent $\eta=4.5$. Further let $\frac{P}{F_{\mathrm{N}} N_{0} B}=117 \mathrm{~dB}$, corresponding for instance to $P=15 \mathrm{dBm}, F_{\mathrm{N}}=5 \mathrm{~dB}, N_{0}=-174 \mathrm{dBm} / \mathrm{Hz}$ and $B=5 \mathrm{MHz}$. In one case, every link has a distance of $d=40 \mathrm{~m}$ whereas, in another case, the link distances equiprobably take the values $d_{1}=20 \mathrm{~m}, d_{2}=40 \mathrm{~m}$ and $d_{3}=60 \mathrm{~m}$. Shown in Table I are the co-channel link densities of ITLinQ type I and type II computed via (15) and (16) for $M=10$ with $\mu=0.5,0.7,0.9$. As $\mu$ increases, $r$ shrinks and hence the link density increases.

\section{Interference Modeling}

To model the interference, we follow the approach in [43]:

1) The short-term (local) distribution of $z_{0}^{\prime}$ is modeled as zero-mean complex Gaussian with matched conditional variance $\sigma_{0}^{2}=\mathbb{E}\left[\left|z_{0}^{\prime}\right|^{2} \mid\left\{r_{v_{0}, x_{k}}, e_{k}\right\}\right]$, where the expectation is over the data and fading distributions. Besides the central limit theorem, there are informationtheoretic arguments in favor of modeling the aggregate interference as complex Gaussian with a power dictated by the locations of the interferers: if the exact distribution of the interference is either unknown or ignored by the receiver, with a decoder designed to handle Gaussian noise, then the achievable spectral efficiency is precisely as if the interference were indeed Gaussian [44].

2) The interfering transmitters within an averaging circle surrounding the receiver of interest are explicitly modeled while the aggregate interference emanating from outside this circle 
is replaced by its expected (over the interferer locations) value. With an averaging circle radius $R=\sqrt{K /(\pi \lambda)}$, the average number of interferer transmitters explicitly modeled is $K$ (cf. Fig. 2). As described in [43], the choice of $K$ should be chosen to balance simplicity and accuracy in capturing the performance for specific user locations.

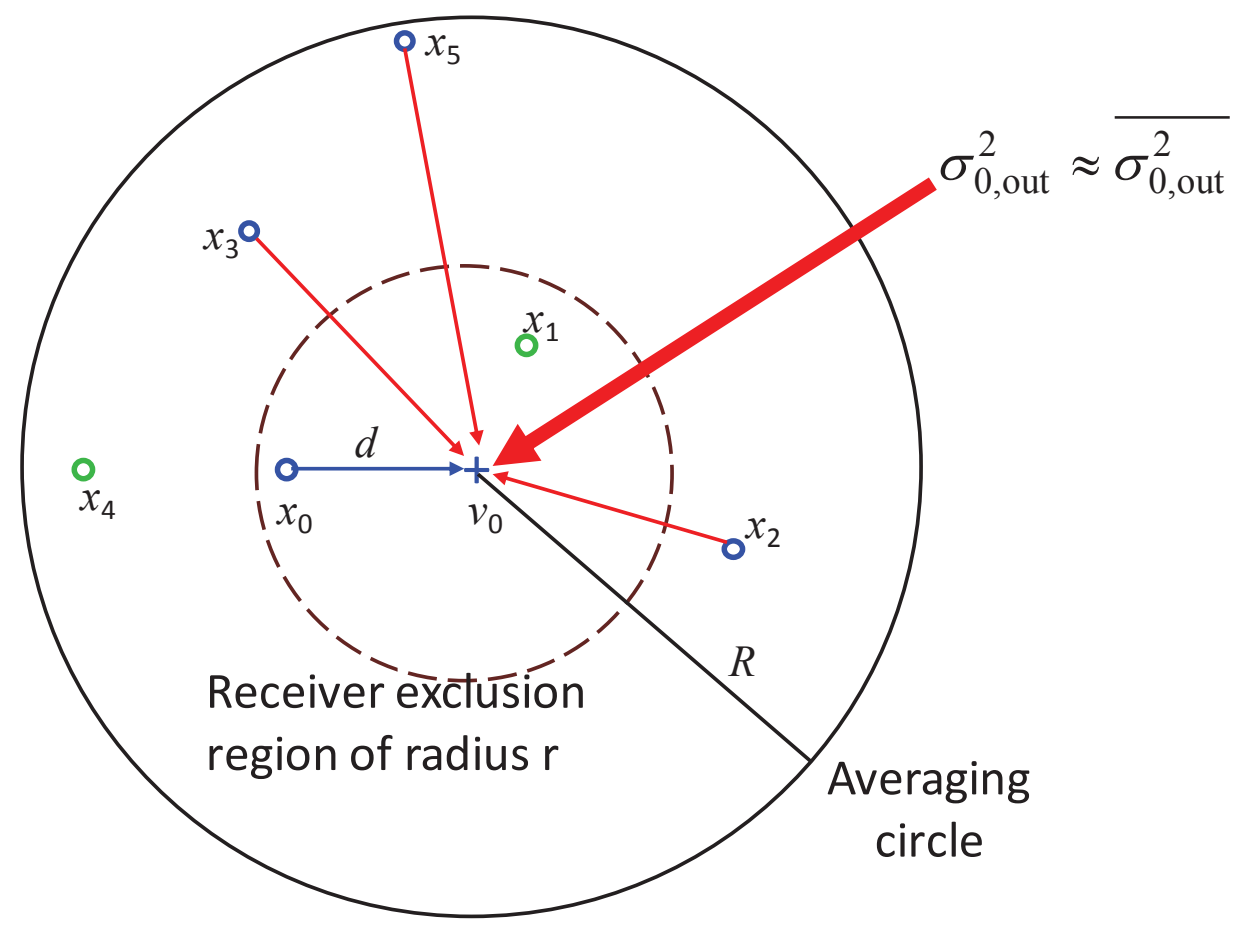

Fig. 2: Interference modeling illustration where the intended link distance is $d$, the receiver exclusion region radius is $\mathrm{r}$, and $K=5$ interferer transmitters with retaining indicators $\left\{e_{k}\right\}_{k=1}^{5}=$ $\{0,1,1,0,1\}$ are explicitly modeled. The aggregate interference $\sigma_{0 \text {,out }}^{2}$ emanating from outside the averaging circle of radius $R$ is replaced by its expectation (over the corresponding interferer locations).

In reference to the first point above: Gaussian noise is the worst-case additive noise in wireless networks [45] and thus it is not only the case that the results obtained under our Gaussian interference model are operationally more relevant than the quantities computed numerically for the non-Gaussian distribution of $z_{0}^{\prime}$ as defined in (3), but also that the former are always (tight) lower bounds to the latter. Recalling the definition in (3), the conditional variance $\sigma_{0}^{2}$ (i.e., the 
power of $z_{0}^{\prime}$ for given interference locations $\left\{r_{v_{0}, x_{k}}\right\}$ and retaining indicators $\left.\left\{e_{k}\right\}\right)$ is

$$
\sigma_{0}^{2}=\underbrace{\sum_{k=1}^{K} P e_{k} r_{v_{0}, x_{k}}^{-\eta}}_{\sigma_{0, \text { in }}^{2}}+\underbrace{\sum_{k=K+1}^{\infty} P e_{k} r_{v_{0}, x_{k}}^{-\eta}+F_{\mathrm{N}} N_{0} B}_{\sigma_{0, \text { out }}^{2}}
$$

where, applying the second point in our interference modelling approach, $\sigma_{0 \text {,in }}^{2}$ corresponds to the $K$ transmitters in $\Phi \cap \mathcal{B}_{v_{0}}(R)$ while $\sigma_{0 \text {,out }}^{2}$ corresponds to the transmitters in $\Phi \backslash \mathcal{B}_{v_{0}}(R)$ plus noise.

Computing the expectation of $\sigma_{0 \text {,out }}^{2}$ over the locations of the interfering transmitters in that term is not straightforward because ITLinQ causes dependent thinning of the initial PPP and hence the locations of co-channel transmitters no longer conform to a PPP. To circumvent this obstacle, we borrow a modelling assumption introduced in [23] and whose goodness for our purposes is validated later in the paper.

Assumption 1: The locations of co-channel transmitters outside the receiver's exclusion region $\mathcal{B}_{v_{0}}(\mathrm{r})$ belong to a homogeneous PPP with scaled-down densities $\lambda_{\Psi_{\mathrm{I}}}$ and $\lambda_{\Psi_{\mathrm{II}}}$ for type I and type II, respectively.

Under Assumption 1, the locations of all interferers are modeled as PPP. Recalling that $K$ dominant interferer locations are explicitly modeled, the expectation of $\sigma_{0 \text {,out }}^{2}$ over this PPP gives

$$
\begin{aligned}
\overline{\sigma_{0, \text { out }}^{2}} & =\mathbb{E}\left[\sum_{k=K+1}^{\infty} P e_{k} r_{v_{0}, x_{k}}^{-\eta}\right]+F_{\mathrm{N}} N_{0} B \\
& \approx \int_{R}^{\infty} 2 \pi \lambda_{\Psi} P r^{1-\eta} \mathrm{d} r+F_{\mathrm{N}} N_{0} B \\
& =\frac{2 \pi \lambda_{\Psi} P}{(\eta-2) R^{\eta-2}}+F_{\mathrm{N}} N_{0} B
\end{aligned}
$$

where (20) follows from Assumption 1 and Campbell's theorem [36].

With the interference thus approximated, and recalling the intended signal term in (2), the instantaneous SINR (signal-to-interference-plus-noise ratio) is itself approximated by

$$
\begin{aligned}
\operatorname{SINR}_{0} & \approx \frac{P r_{v_{0}, x_{0}}^{-\eta} \mathbb{E}\left[\left|h_{v_{0}, x_{0}} s_{0}\right|^{2} \mid h_{v_{0}, x_{0}}\right]}{\sigma_{0, \text { in }}^{2}+\overline{\sigma_{0, \text { out }}^{2}}} \\
& =\rho_{0}\left|h_{v_{0}, x_{0}}\right|^{2}
\end{aligned}
$$

where

$$
\rho_{0}=\frac{P r_{v_{0}, x_{0}}^{-\eta}}{\sum_{k=1}^{K} P e_{k} r_{v_{0}, x_{k}}^{-\eta}+\frac{2 \pi \lambda_{\Psi} P}{(\eta-2) R^{\eta-2}}+F_{\mathrm{N}} N_{0} B}
$$

is the (approximate) local-average SINR at the receiver. 


\section{E. SINR Distribution}

For a specific channelized network realization, i.e., given $\left\{r_{v_{0}, x_{k}}, e_{k}\right\}_{k=1}^{K}$, the value of $\rho_{0}$ becomes determined. Since $\left|h_{v_{0}, x_{0}}\right|^{2}$ is exponentially distributed with unit mean, it follows from (23) that the SINR exhibits an exponential distribution with mean $\rho_{0}$ and thus its conditional $\mathrm{CDF}$ is

$$
F_{\operatorname{SINR}_{0} \mid \rho_{0}}(\gamma)=1-e^{-\gamma / \rho_{0}}
$$

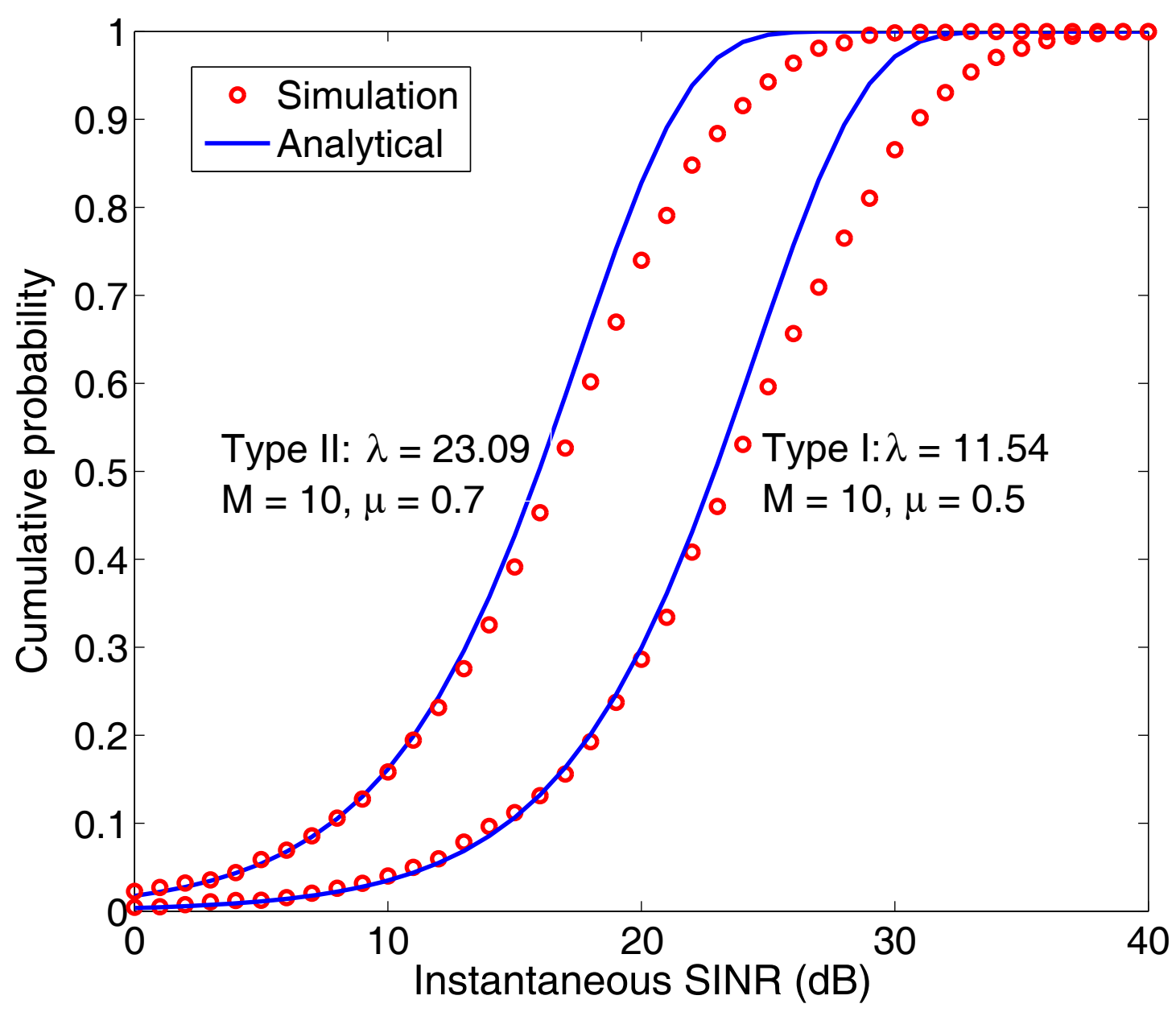

Fig. 3: CDF of instantaneous SINR with fixed link distance $d=40 \mathrm{~m}$.

Example 2. Reconsider Example 1 with the same distance $d=40 \mathrm{~m}$ for each link. The number of explicitly modelled interferers is set to $K=5$ and, to render the system as typical as possible, 
$r_{v_{0}, x_{k}}$ for $k=1, \ldots, K$ is set to the expected distance to the $k$ th nearest neighboring point in a PPP with density $\lambda$ [46]. This gives $r_{v_{0}, x_{k}}=\Gamma(0.5+k) /(\sqrt{\pi \lambda} \Gamma(k))$ where $\Gamma(\cdot)$ is the Gamma function. Shown in Fig. 3 are the SINR distributions for ITLinQ types I and II obtained analytically under Assumption 1 (Eqs. 24 and 25) alongside the simulated distributions with $z_{0}^{\prime}$ as per (3), i.e., without any approximations. In the simulations, the locations of interferers outside the averaging circle no longer conform to a PPP, as the interferers within the exclusion regions are deactivated.

A satisfactory agreement is observed for both ITLinQ types, supporting the validity of our interference modeling approach. Similar agreement is observed for other values of the parameters.

\section{F. Spectral Efficiency}

Having validated our interference modeling approach in the previous subsection, we now turn our attention to the ergodic spectral efficiency, arguably the most operationally relevant quantity in contemporary systems [47].

1) Specific Network Geometry: For a specific network realization, i.e., for a given $\rho_{0}$, the ergodic spectral efficiency of the 0 th link is

$$
\begin{aligned}
C\left(\rho_{0}\right) & =\int_{0}^{\infty} \log _{2}(1+\gamma) \mathrm{d} F_{\text {SINR }_{0} \mid \rho_{0}}(\gamma) \\
& =e^{1 / \rho_{0}} E_{1}\left(\frac{1}{\rho_{0}}\right) \log _{2} e
\end{aligned}
$$

where $E_{1}(\zeta)=\int_{1}^{\infty} t^{-1} e^{-\zeta t} \mathrm{~d} t$ is an exponential integral.

2) Average Network Geometry: Next, we average the link spectral efficiency $C(\cdot)$ over all possible network realizations, obtaining the central result in the paper.

Proposition 1. For transmitter density $\lambda$, the link distance distribution in (1) and parameters $M$ and $\mu$, the spatially averaged link spectral efficiency $(\mathrm{b} / \mathrm{s} / \mathrm{Hz}$ per link) of ITLinQ equals

$$
\begin{aligned}
\bar{C}(\lambda, M, \mu)=\sum_{n=1}^{N} q_{n} \int_{0}^{\infty} \frac{\log _{2} e}{\gamma+1} \exp \{ & -\frac{\gamma}{d_{n}^{-\eta}} \frac{N_{0} F_{\mathrm{N}} B}{P}+\pi \lambda_{\Psi} \mathrm{r}_{n}^{2} \\
& \left.+\frac{2}{\eta} \pi \lambda_{\Psi} \gamma^{\frac{2}{\eta}} d_{n}^{2} \bar{\Gamma}\left(-\frac{2}{\eta}, \gamma\left(\frac{d_{n}}{\mathrm{r}_{n}}\right)^{\eta}\right)\right\} \mathrm{d} \gamma
\end{aligned}
$$

where $q_{n}$ is the fraction of co-channel links with distance $d_{n}$ and $\lambda_{\Psi}$ depends on $\lambda, M$ and $\mu$ as per (15) for type I and (16) for type II, while $\bar{\Gamma}(\cdot, \cdot)$ is the lower incomplete Gamma function.

Proof. Appendix B. 


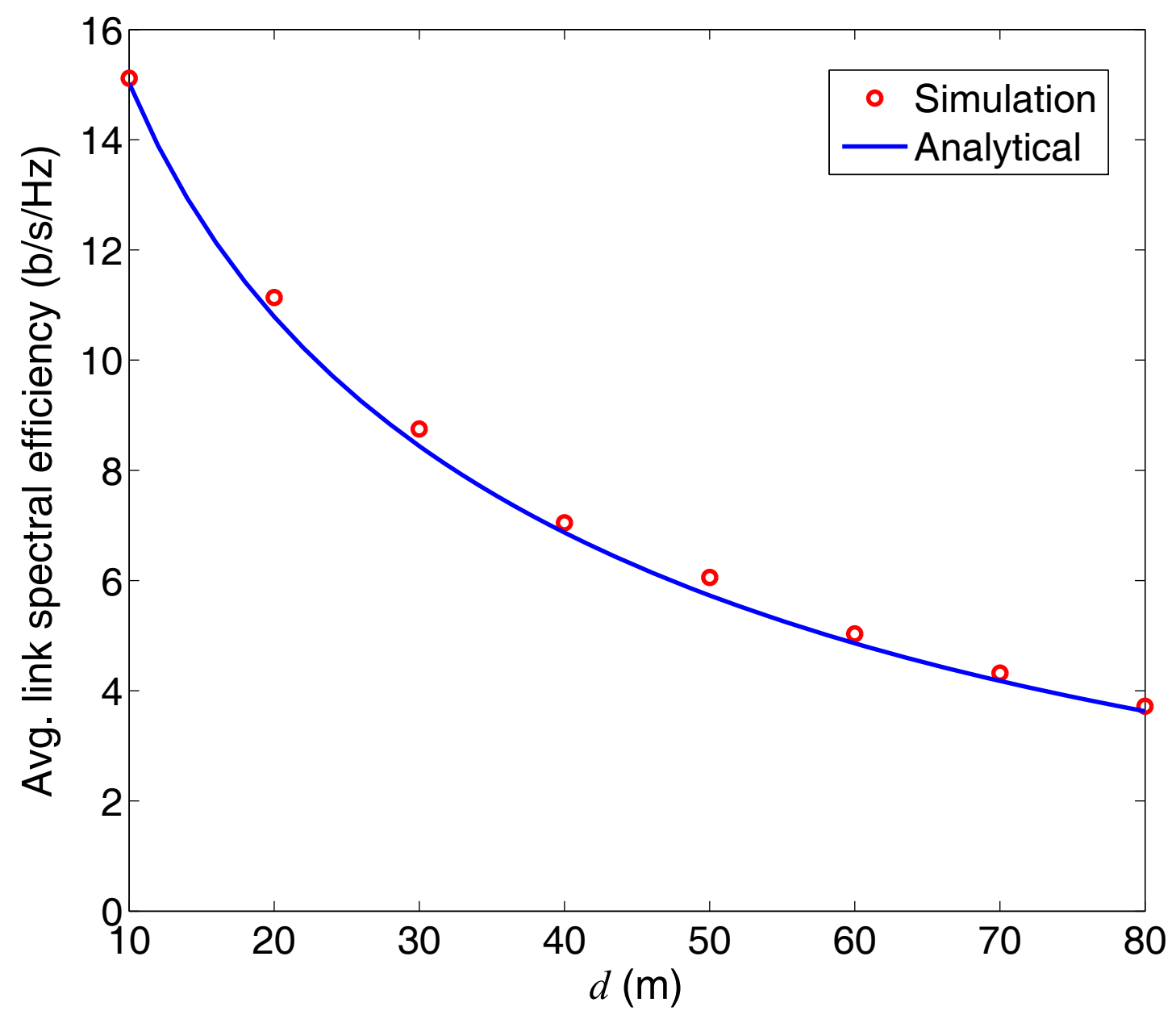

Fig. 4: Average link spectral efficiency of ITLinQ type II as function of $d$ for $\lambda=11.54$ links $/ \mathrm{km}^{2}, M=10$ and $\mu=0.7$.

Example 3. Shown in Fig. 4 is a comparison of $\bar{C}(\lambda, M, \mu)$ as given in Proposition 1 against its simulated counterpart for $\lambda=11.54$ links $/ \mathrm{km}^{2}, \eta=4.5$ and $\frac{P}{F_{\mathrm{N}} N_{0} B}=117 \mathrm{~dB}$. Every link has the same intended distance $d$. The channelization is ITLinQ type II with $M=10$ and $\mu=0.7$. The simulated result corresponds to the exact mutual information under the non-Gaussian interference in (3), computed through Monte-Carlo histograms and averaged over many fading realizations and interference locations. The match is excellent, again evincing the goodness of our interference modeling approach. And, as anticipated earlier, the results of our analysis are slightly below the mutual information computed under the non-Gaussian interference in (3). 
From Proposition 1, by taking $\lim _{M \rightarrow \infty} \bar{C}(\lambda, M, \mu)$, we can recover the spatially averaged link spectral efficiency for an unchannelized network, i.e., a network where all links are co-channel. This baseline,

$$
\bar{C}(\lambda, \infty, \mu)=\sum_{n=1}^{N} p_{n} \int_{0}^{\infty} \frac{\log _{2} e}{\gamma+1} \exp \left\{-\frac{\gamma}{d_{n}^{-\eta}} \frac{F_{\mathrm{N}} N_{0} B}{P}+\pi \lambda \gamma^{\frac{2}{\eta}} d_{n}^{2} \Gamma\left(1-\frac{2}{\eta}\right)\right\} \mathrm{d} \gamma
$$

shall be useful to establish the gains of ITLinQ.

Furthermore, from Proposition 1 we can also obtain the spatially averaged system spectral efficiency (b/s/Hz per unit area) by scaling the spatially averaged link spectral efficiency $\bar{C}(\lambda, M, \mu)$ by the co-channel link density $\lambda_{\Psi}$. This gives

$$
\overline{\mathcal{C}}(\lambda, M, \mu)=\lambda_{\Psi} \bar{C}(\lambda, M, \mu)
$$

from which the average benefits of channelization over all possible network geometries can be gauged. Moreover, the parameters $M$ and $\mu$ can be tuned as function of the user density and link distance distribution in order to maximize the average system spectral efficiency, i.e., to obtain

$$
\overline{\mathcal{C}}^{\star}(\lambda)=\max _{M>0, \mu>0} \overline{\mathcal{C}}(\lambda, M, \mu)
$$

Example 4. Shown in Fig. 5 is the average system spectral efficiencies of ITLinQ type I and type II obtained by numerically solving (31) with link distances fixed at either $d=20 \mathrm{~m}$ or $d=40 \mathrm{~m}$. Type II is seen to be uniformly superior, with a performance advantage that increases with the user density, and hence we concentrate on this type henceforth. The limiting $(\lambda \rightarrow \infty)$ system spectral efficiency of ITLinQ type II is presented in Fig. 6 as function of the link distance $d$, fixed for all users.

We observe that, for any fixed intended link distance, the co-channel link density $\lambda_{\Psi_{I I}}$ increases with growing $\lambda$ and eventually saturates. This, in turn, leads to saturation in the average system spectral efficiency.

\section{FlashLinQ}

In addition to the baseline of a network with no channelization, a second interesting baseline for ITLinQ is FlashLinQ. In this section we briefly review how FlashLinQ operates, couching it in our notation and molding it to our models. 


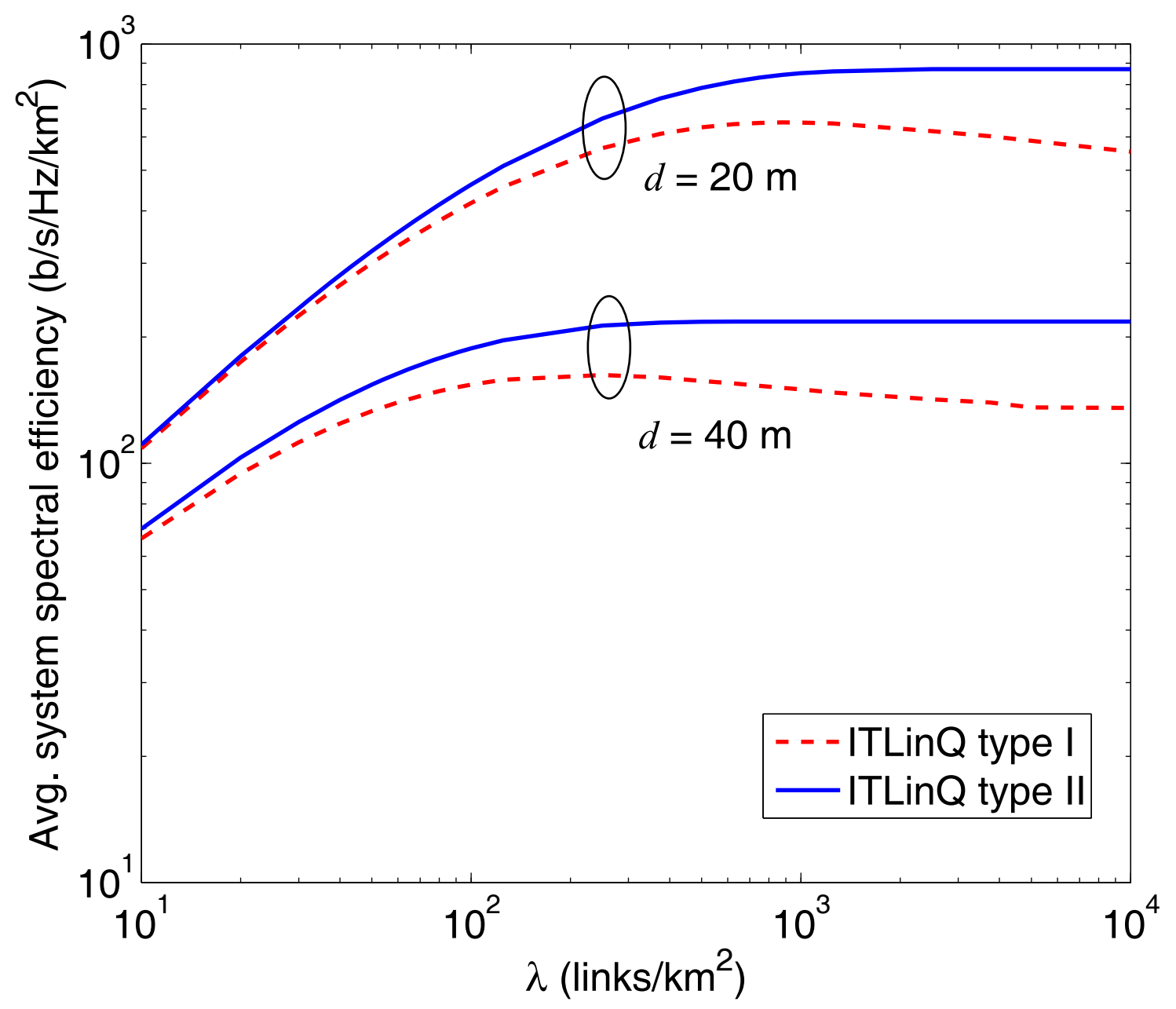

Fig. 5: Average system spectral efficiency v. $\lambda$ for optimized ITLinQ with $d=20 \mathrm{~m}$ and $d=40$ $\mathrm{m}$, and with $\eta=4.5$ and $\frac{P}{F_{\mathrm{N}} N_{0} B}=117 \mathrm{~dB}$.

\section{A. Description}

In contrast with ITLinQ, whose channelization conditions are grounded in an informationtheoretic optimality notion and then heuristically refined, FlashLinQ's channelization policy was formulated on a heuristic basis from the onset.

For the sake of consistency, and to ensure a fair comparison later, we consider a type II embodiment of FlashLinQ for which the 0th link is allowed in a channel if and only if two distinct conditions are satisfied with respect to links with a lower mark. The first condition is

$$
\frac{\mathrm{SNR}}{\operatorname{INR}_{i, 0}^{\text {out }}} \geq \gamma_{\mathrm{TX}} \quad i \in\left\{n: m_{n}<m_{0}\right\}
$$




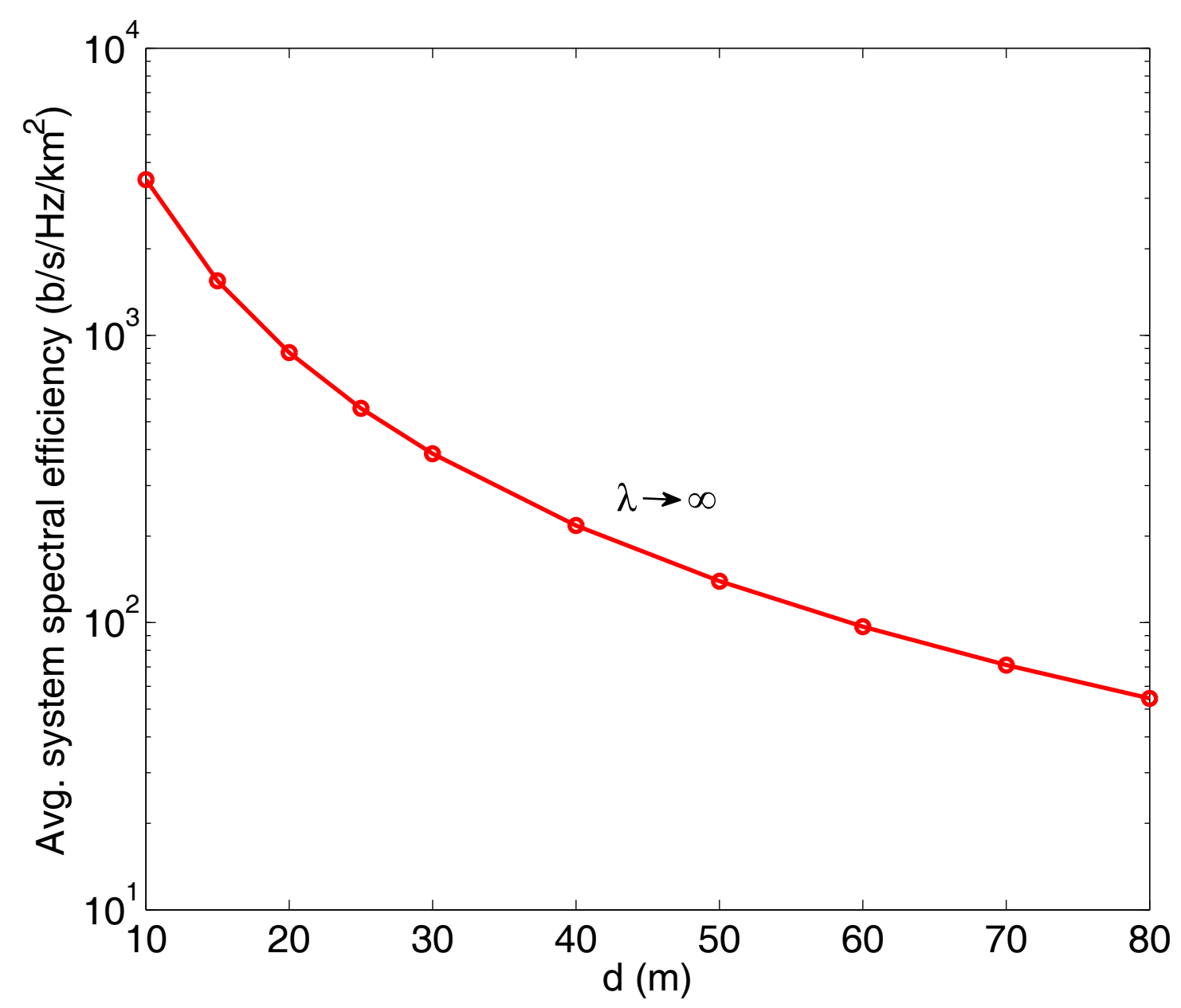

Fig. 6: Average system spectral efficiency v. $d$ for optimized ITLinQ type II with $\lambda \rightarrow \infty$, $\eta=4.5$ and $\frac{P}{F_{\mathrm{N}} N_{0} B}=117 \mathrm{~dB}$.

which ensures that the outgoing interference caused by the 0th-link transmitter to any lower-mark receiver is within a specified limit determined by the threshold $\gamma_{\mathrm{TX}}$; if (32) is not satisfied, then the 0th-link transmitter must yield, meaning that it must refrain from transmitting and thus the link must be allocated to another channel. In turn, the second condition is

$$
\frac{\mathrm{SNR}}{\sum_{k} \mathrm{INR}_{0, k}^{\mathrm{in}}} \geq \gamma_{\mathrm{RX}} \quad k \in\left\{n: m_{n}<m_{0}\right\}
$$

where $\gamma_{\mathrm{Rx}}$ is an additional threshold; if (33) is not satisfied, then the 0th-link receiver must yield, meaning again that the link must be allocated to another channel. 


\section{B. Geometric Interpretation}

Paraphrasing [31], the condition in (32) intends for the 0th link not to cause too much interference to other lower-mark links assigned to the channel under consideration. However, no specific SIR can be guaranteed for those other links as the condition limits the interference contribution of the 0th link without regard to how much other interference is present. Plugging into (32) the definitions of SNR and INR ${ }_{i, 0}^{\text {out }}$ given in (4) and (7), respectively, we obtain for the $i$ th lower-mark interfered link the equivalent condition

$$
r_{v_{i}, x_{0}} \geq r_{v_{0}, x_{0}} \gamma_{\mathrm{TX}}^{1 / \eta}
$$

whose enforcement amounts to forming around the transmitter a circular exclusion region that is free of any lower-mark receiver and whose radius is

$$
r_{v_{0}, x_{0}} \gamma_{\mathrm{TX}}^{1 / \eta}
$$

Contrasting this definition with ITLinQ's exclusion radius $r$ (cf. Eq. 14), we observe that the two coincide if $\mu=1$ and $M=1 / \gamma_{\mathrm{TX}}$.

Given that the first FlashLinQ condition by itself cannot prevent possible situations of excessive interference, it is reinforced by the second condition meant to help achieve, again paraphrasing [31], a reasonable SIR for the 0th link. Once more though, no specific value can be guaranteed for the SIR because the second condition too involves only links with lower marks; the interference from higher-mark links may push the SIR below $\gamma_{R x}$ and, with some small probability, below even lower values. Furthermore, because the second condition involves not the interference from a specific link but a sum thereof, geometrically it amounts to forming an exclusion region thateven in the absence of shadowing - is not circular; this renders FlashLinQ's analysis rather unwieldy.

\section{Average Spectral Efficiency}

In the original formulation of FlashLinQ, fixed values were employed for $\gamma_{\mathrm{TX}}$ and $\gamma_{\mathrm{RX}}$ with the further restriction that $\gamma_{\mathrm{TX}}=\gamma_{\mathrm{RX}}$. Then, in [48], this restriction was lifted and the performance of FlashLinQ was numerically computed for varying threshold values to find that:

- The average system spectral efficiency decreases with growing $\gamma_{\mathrm{TX}}$.

- The average system spectral efficiency increases with growing $\gamma_{\mathrm{Rx}}$ for lower values of $\gamma_{\mathrm{Rx}}$ and then gradually decreases with growing $\gamma_{\mathrm{Rx}}$ for higher values of $\gamma_{\mathrm{Rx}}$. 


\section{Performance Evaluation of ITLinQ}

With the theoretical framework established in Section III, we now proceed to evaluate the performance of optimized ITLinQ type II, and contrast it with the unchannelized baseline and with FlashLinQ.

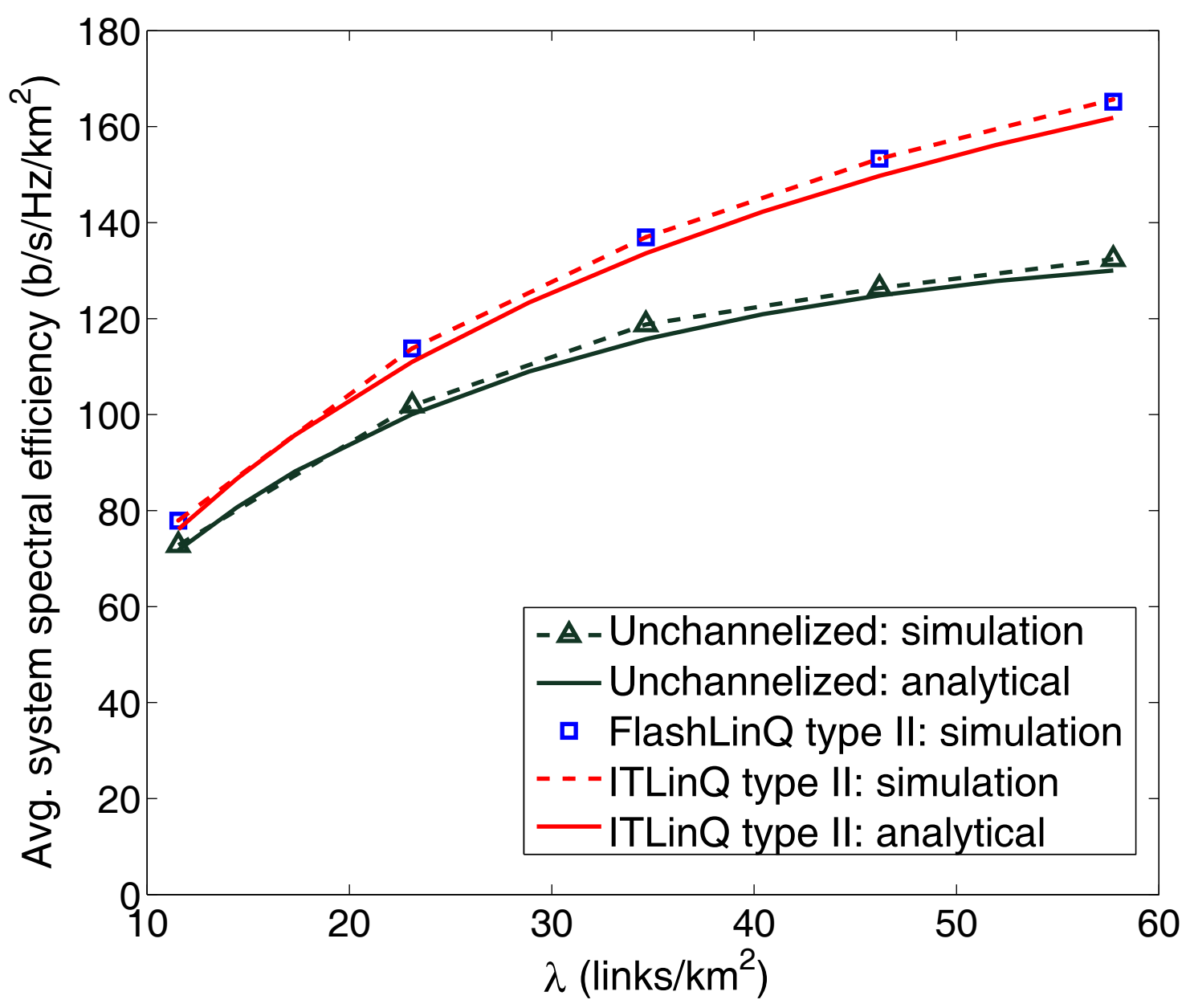

Fig. 7: Average system spectral efficiency v. $\lambda$ for optimized ITLinQ with $d=40, \eta=4.5$ and $\frac{P}{F_{\mathrm{N}} N_{0} B}=117 \mathrm{~dB}$. Also shown are the corresponding spectral efficiencies for FlashLinQ and for an unchannelized network.

Example 5. Fig. 7 shows, as function of $\lambda$, the average system spectral efficiency of optimized ITLinQ when every link has an intended distance $d=40 \mathrm{~m}$. Both analytical and simulation results are provided. As baselines, analytical and simulation results for an unchannelized network, as well as simulation results for FlashLinQ. 


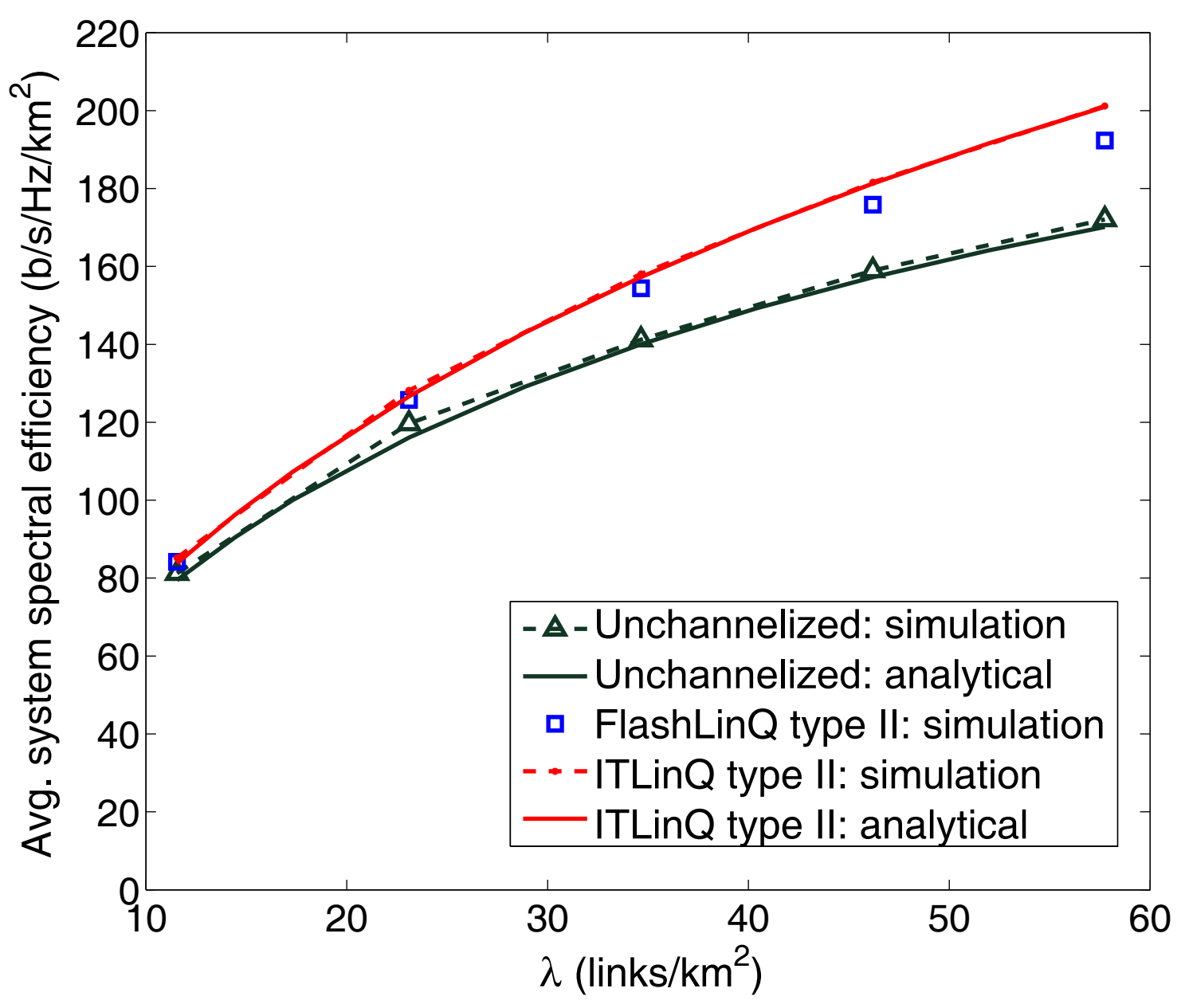

Fig. 8: Average system spectral efficiency v. $\lambda$ for optimized ITLinQ with randomized link distances (equiprobably: $d_{1}=20 \mathrm{~m}, d_{2}=40 \mathrm{~m}$ and $d_{2}=60 \mathrm{~m}$ ) and with $\eta=4.5$ and $\frac{P}{F_{\mathrm{N}} N_{0} B}=117 \mathrm{~dB}$. Also shown are the corresponding spectral efficiencies for FlashLinQ and for an unchannelized network.

Example 6. Fig. 8 shows, as function of $\lambda$, the average system spectral efficiency of optimized ITLinQ when the link distances equiprobably take the values $d_{1}=20 \mathrm{~m}, d_{2}=40 \mathrm{~m}$ and $d_{2}=60$ m. Again, as baselines we include analytical and simulation results for an unchannelized network as well as simulation results for FlashLinQ.

From the foregoing examples, we can draw the following observations:

- Despite the PPP approximation in Assumption 1, the analytical expressions derived for ITLinQ are very accurate—slightly conservative for the reasons exposed in Section III-D- 
and offer an efficient alternative to simulations. Indeed, the generation of each optimized simulation point in Examples 5 and 6 is an extremely time-consuming process.

- Both ITLinQ and FlashLinQ yield a significant improvement (on the order of 20-30\% according to the foregoing examples) in average system spectral efficiency relative to the unchannelized baseline.

- ITLinQ has a slight edge over FlashLinQ, which despite its fully heuristic nature performs remarkably well if its thresholds are properly optimized.

Our observations have been verified to hold qualitatively for other link distance distributions, for pathloss exponents ranging between $\eta=3$ and $\eta=4.5$, for dual-slope pathloss functions [49], and even with shadow fading incorporated.

While ITLinQ's improvement in average system spectral efficiency with respect to an unchannelized network is on the range of 20-30\%, much more sizable gains are observed by users suffering from above-average interference. We next illustrate this potential in a typical situation where strong interference arises.

Example 7. Consider $\lambda=23.09$ links $/ \mathrm{km}^{2}$, which amounts to an average of $20 \mathrm{D} 2 \mathrm{D}$ users per cell of radius $525 \mathrm{~m}$, with the same distance $d=40 \mathrm{~m}$ for each link. As in earlier examples,

$\eta=4.5$ and $\frac{P}{F_{\mathrm{N}} N_{0} B}=117 \mathrm{~dB}$. The parameters of ITLinQ are set to maximize the average system spectral efficiency, i.e., $M=10$ and $\mu=0.5$, corresponding to an exclusion radius of $r=75.6 \mathrm{~m}$. Shown in Fig. 9 are the distributions of system spectral efficiency for ITLinQ and for unchannelized network. ITLinQ exhibits a hefty advantage in the lower tail of the distribution, specifically a seven-fold gain in terms of the system spectral efficiency achieved by the worst $10 \%$ of network geometries. (The gain shrinks progressively as higher portions of the CDF are considered and, for the most favorable network geometries, ITLinQ is altogether unnecessary.)

\section{CONCLUSION}

The availability of multiple channels in D2D networks offers the possibility of parsing the available links onto various sets in such a way that excessive interference is avoided. ITLinQ and FlashLinQ operate by enforcing - through various parameters and thresholds-exclusion regions around transmitters and receivers. Both are effective, with a slight edge for ITLinQ. With respect to an unchannelized network, the gains in system spectral efficiency range between $20-30 \%$ on average and multiple-fold improvement factors on the most unfavorable network geometries. 


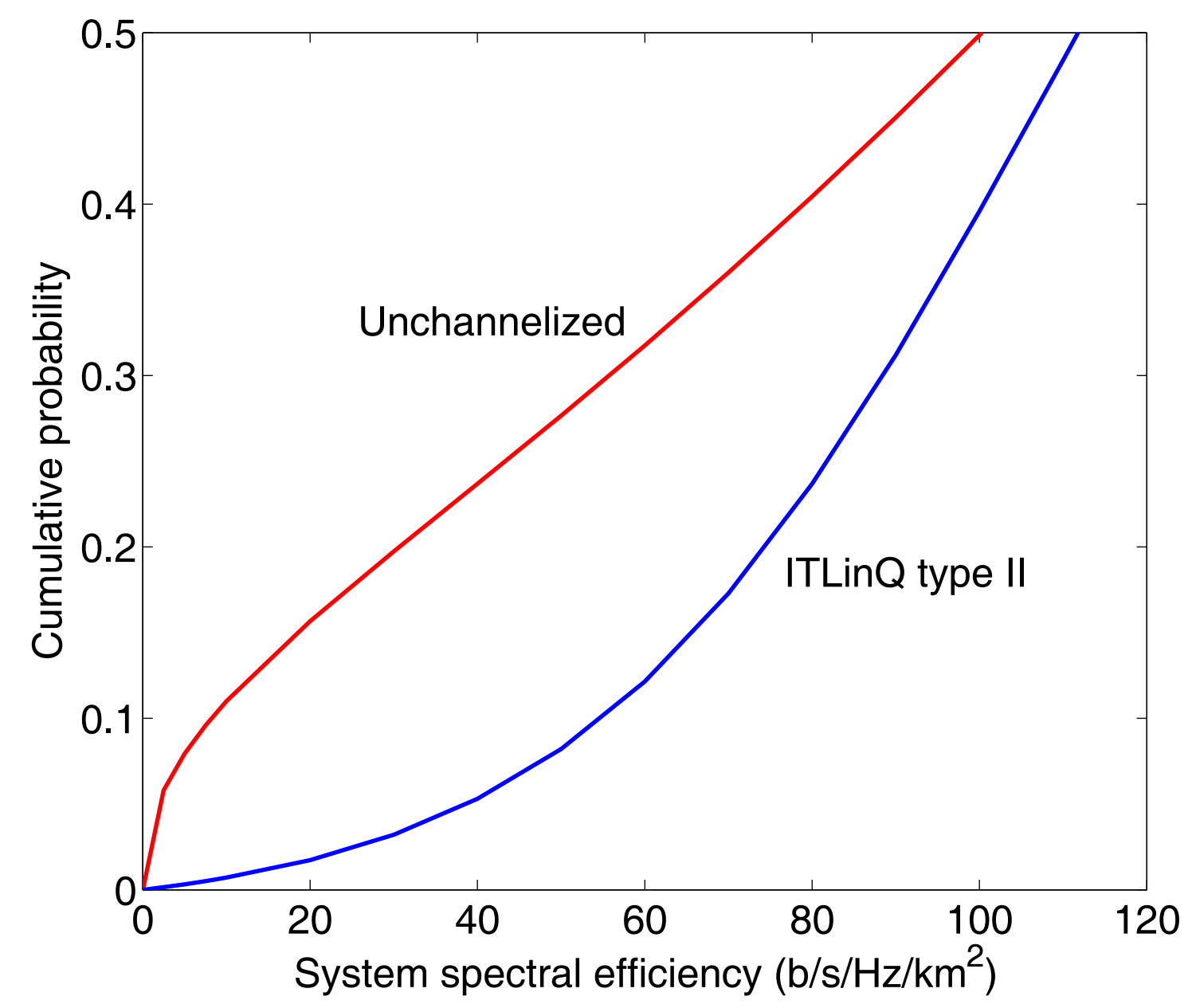

Fig. 9: CDF of system spectral efficiency with intended link distances $d=40 \mathrm{~m}$.

Borrowing techniques from [21], [22], the analysis could be extended to incorporate directional antennas and sectorization; while quantitatively very interesting, this extension is not expected to modify the qualitative conclusions.

\section{ACKNOWLEDGMENT}

The authors gratefully acknowledge the comments and the ITLinQ simulation software provided by Navid Naderi Alizadeh, from the University of Southern California. 


\section{APPENDIX A}

\section{PROOF OF LEMMA 1}

The density of a stationary point process is defined as the ratio of the expected number of points in an arbitrary region $B$ to the Lebesgue measure of that region. Using this definition, the density of ITLinQ type I can be written as

$$
\begin{aligned}
\lambda_{\Psi_{\mathrm{I}}} & =\frac{1}{v(B)} \mathbb{E}\left[\sum_{x_{k} \in \Psi_{\mathrm{I}}} \mathbb{1}\left(x_{k} \in B\right)\right] \\
& =\frac{1}{v(B)} \mathbb{E}\left[\sum_{x_{k} \in \Phi} \mathbb{1}\left(e_{k}=1\right) \mathbb{1}\left(x_{k} \in B\right)\right]
\end{aligned}
$$

where $v(B)$ is the Lebesgue measure of $B$ and (37) holds due to the construction of $\Psi_{\mathrm{I}}$, i.e., the process of co-channel transmitter locations for ITLinQ type I. Expanding the expectation in (37) by means of the notion of Palm distribution or measure [36], we obtain

$$
\begin{aligned}
\mathbb{E}\left[\sum_{x_{k} \in \Phi} \mathbb{1}\left(e_{k}=1\right) \mathbb{1}\left(x_{k} \in B\right)\right] & =\int_{\hat{\mathcal{N}}} \sum_{x_{k} \in \phi \cap B} \mathbb{1}\left(\hat{\phi} \in V_{x_{k}}\right) \hat{\mathrm{P}}(\mathrm{d} \hat{\phi}) \\
& =\lambda v(B) \hat{\mathrm{P}}_{x_{0}}(V)
\end{aligned}
$$

where (39) follows from the definition of Palm distribution of a marked PPP [36] while $\phi$ and $\hat{\phi}$ are realizations of $\Phi$ and $\hat{\Phi}$, respectively, $\hat{\mathcal{N}}$ is the sample space of $\hat{\Phi}$ and $\hat{\mathrm{P}}_{x_{0}}(V)$ is the Palm

distribution of a stationary marked point process $\hat{\Phi}$ with a transmitter location conditioned at $x_{0}$ and with $V$ the property of $\hat{\Psi}_{\mathrm{I}}$,

$$
\begin{aligned}
V & =V_{x_{0}} \\
& =\left\{\left\{x_{k}\right\}_{k=1}^{\infty} \cap \mathcal{B}_{v_{0}}(\mathrm{r})=\varnothing\right\} \cap\left\{\left\{v_{i}\right\}_{i=1}^{\infty} \cap \mathcal{B}_{x_{0}}(\mathrm{r})=\varnothing\right\}
\end{aligned}
$$

which represents the channelization condition imposed by ITLinQ type I (cf. Sec. III-A), with a location conditioned at $x_{0}$.

Combining (37) and (39),

$$
\begin{aligned}
\lambda_{\Psi_{\mathrm{I}}} & =\lambda \hat{\mathrm{P}}_{x_{0}}(V) \\
& =\lambda \mathbb{P}\left(\hat{\Phi} \in V \mid x_{0} \in \Phi\right) \\
& =\lambda \sum_{n=1}^{N} p_{n} \mathbb{P}\left(\hat{\Phi} \in V \mid x_{0} \in \Phi, r_{v_{0}, x_{0}}=d_{n}\right)
\end{aligned}
$$


where (43) follows from expressing the Palm distribution $\hat{\mathrm{P}}_{x_{0}}(V)$ in terms of the Palm probability [36], [50], with $\hat{\Phi} \in V$ meaning that $\hat{\Phi}$ has property $V$. Recalling (41), it can be seen that $\mathbb{P}\left(\hat{\Phi} \in V \mid x_{0} \in \Phi, r_{v_{0}, x_{0}}=d_{n}\right)$ in (44) is a function of $(i)$ the probability that there exists no co-channel transmitter in the receiver exclusion circle $\mathcal{B}_{v_{0}}\left(\mathrm{r}_{n}\right)$, and (ii) the probability that there exists no co-channel receiver in the transmitter exclusion circle $\mathcal{B}_{x_{0}}\left(r_{n}\right)$. Then, conditioned on $r_{v_{0}, x_{0}}=d_{n}$, we have

$$
\begin{aligned}
\mathbb{P}\left(\hat{\Phi} \in V \mid x_{0} \in \Phi\right)= & \left.\mathbb{P}\left(\left\{\left\{x_{k}\right\}_{k=1}^{\infty} \cap \mathcal{B}_{v_{0}}\left(\mathrm{r}_{n}\right)=\varnothing\right\} \cap\left\{\left\{v_{i}\right\}_{i=1}^{\infty} \cap \mathcal{B}_{x_{0}}(\mathrm{r})=\varnothing\right\}\right\}\right) \\
= & \mathbb{P}\left(\left\{v_{i}\right\}_{i=1}^{\infty} \cap \mathcal{B}_{x_{0}}\left(\mathrm{r}_{n}\right)=\varnothing \mid\left\{x_{k}\right\}_{k=1}^{\infty} \cap \mathcal{B}_{v_{0}}\left(\mathrm{r}_{n}\right)=\varnothing\right) \\
& \times \mathbb{P}\left(\left\{x_{k}\right\}_{k=1}^{\infty} \cap \mathcal{B}_{v_{0}}\left(\mathrm{r}_{n}\right)=\varnothing\right)
\end{aligned}
$$

We can affirm that

$$
\mathbb{P}\left(\left\{x_{k}\right\}_{k=1}^{\infty} \cap \mathcal{B}_{v_{0}}\left(r_{n}\right)=\varnothing\right)=e^{-\pi \lambda r_{n}^{2}}
$$

is the probability that, within a given area, there exists no point of the PPP [36], [50]. Considering Fig. 10, which illustrates the geometry of the transmitters and receivers of link 0 and link $i$, we can express $\mathbb{P}\left(\left\{v_{i}\right\}_{i=1}^{\infty} \cap \mathcal{B}_{x_{0}}\left(\mathrm{r}_{n}\right)=\varnothing\right)$, conditioned on $r_{v_{0}, x_{0}}=d_{n}$ and $\left\{\left\{x_{k}\right\}_{k=1}^{\infty} \cap \mathcal{B}_{v_{0}}\left(\mathrm{r}_{n}\right)=\varnothing\right\}$, as

$$
\begin{aligned}
\mathbb{P}\left(\left\{v_{i}\right\}_{i=1}^{\infty} \cap \mathcal{B}_{x_{0}}\left(\mathrm{r}_{n}\right)=\varnothing\right) & =\mathbb{P}\left(\bigcap_{x_{i} \in \Phi}\left\{v_{i} \notin \mathcal{B}_{x_{0}}\left(\mathrm{r}_{n}\right)\right\}\right) \\
& =\mathbb{E}\left[\prod_{x_{i} \in \Phi} \mathbb{1}\left(v_{i} \notin \mathcal{B}_{x_{0}}\left(\mathrm{r}_{n}\right)\right)\right] \\
& =\mathbb{E}\left[\prod_{x_{i} \in \Phi} \mathbb{E}\left[\mathbb{1}\left(v_{i} \notin \mathcal{B}_{x_{0}}\left(\mathrm{r}_{n}\right)\right) \mid x_{i}\right]\right] \\
& =\mathbb{E}\left[\prod_{x_{i} \in \Phi} \mathbb{P}\left(v_{i} \notin \mathcal{B}_{x_{0}}\left(\mathrm{r}_{n}\right) \mid x_{i}\right)\right] \\
& =\prod_{\ell=1}^{N}\left[\prod_{x_{i} \in \Phi_{\ell}} \mathbb{P}\left(v_{i} \notin \mathcal{B}_{x_{0}}\left(\mathrm{r}_{n}\right) \mid x_{i}\right)\right] \\
& =e^{-\lambda \sum_{\ell=1}^{N} p_{\ell} \int\left(1-\mathbb{P}\left(v_{i} \notin \mathcal{B}_{x_{0}}\left(\mathrm{r}_{n}\right) \mid x_{i}, r_{v_{i}, x_{i}}=d_{\ell}\right)\right) \mathrm{d} x_{i}}
\end{aligned}
$$

where $\Phi_{\ell}$ in (52) represents the process of transmitters whose intended link distance is $d_{\ell}$ and (53) follows from the PGFL (probability generating functional) of the PPP $\Phi$ with density $\lambda$; the circular region $\mathcal{B}_{v_{0}}\left(r_{n}\right)$ (the shaded circular region in Fig. 10) is excluded from the integration limit in (53) because the transmitters belonging to that region are already deactivated. 


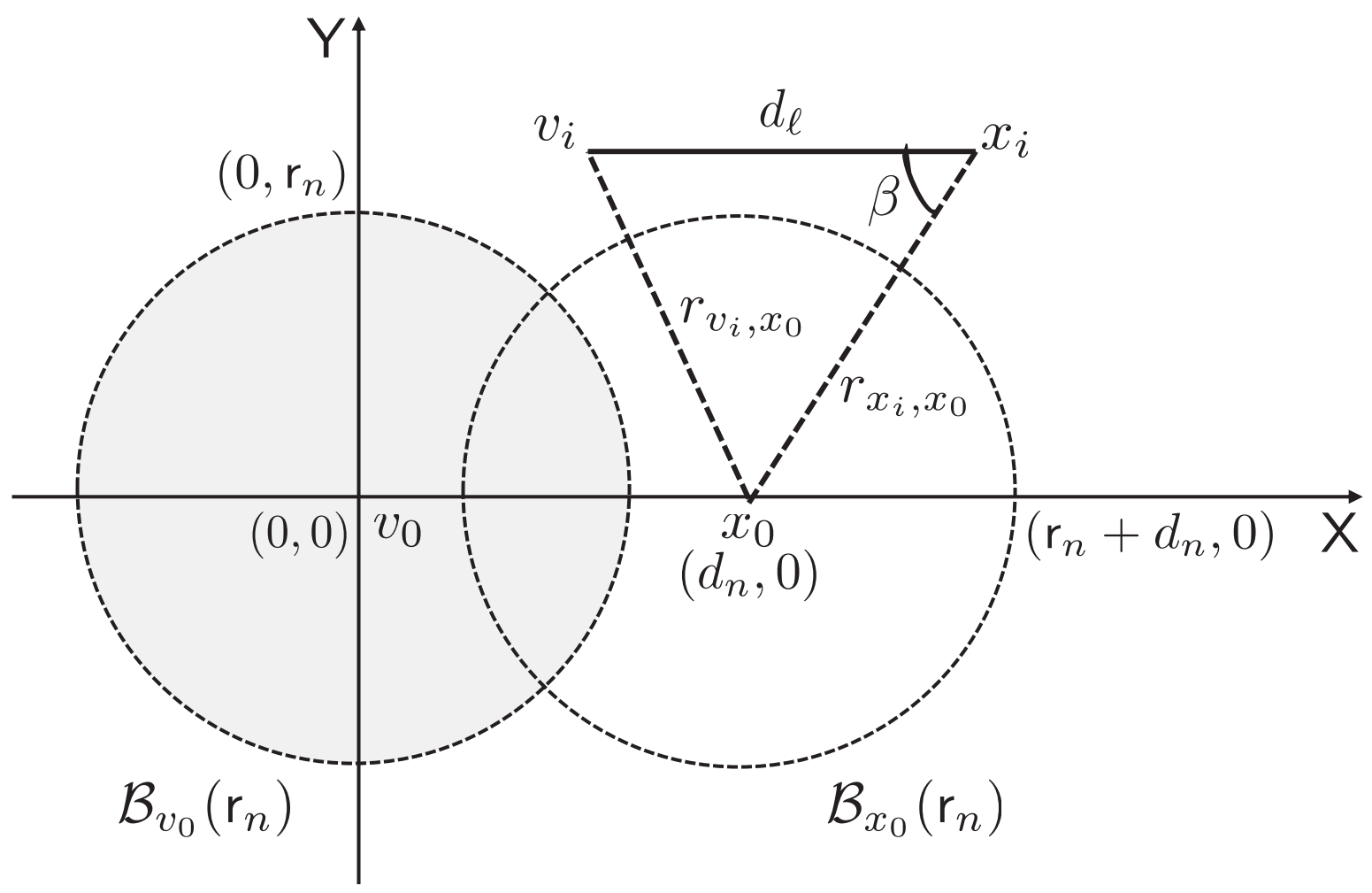

Fig. 10: Illustration of two transmitter-receiver links.

As can be seen in Fig. 10, the probability $\mathbb{P}\left(v_{i} \notin \mathcal{B}_{x_{0}}(\mathrm{r}) \mid x_{i}, r_{v_{i}, x_{i}}=d_{\ell}\right)$ is completely characterized by the distance from the $i$ th link transmitter to the 0th link transmitter $r_{x_{i}, x_{0}}$. First, for $0<r_{x_{i}, x_{0}} \leq\left|\mathrm{r}_{n}-d_{\ell}\right|, \mathbb{P}\left(v_{i} \notin \mathcal{B}_{x_{0}}\left(\mathrm{r}_{n}\right) \mid x_{i}, r_{v_{i}, x_{i}}=d_{\ell}\right)=\mathbb{1}\left(\mathrm{r}_{n}<d_{\ell}\right)$. Next, by applying the law of cosines to the triangle $x_{0} x_{i} v_{i}$ in Fig. 10, we can write

$$
r_{v_{i}, x_{0}}^{2}=r_{x_{i}, x_{0}}^{2}+d_{\ell}^{2}-2 r_{x_{i}, x_{0}} d_{\ell} \cos (\beta)
$$

Then, for $\left|\mathrm{r}_{n}-d_{\ell}\right|<r_{x_{i}, x_{0}} \leq \mathrm{r}_{n}+d_{\ell}$, we can compute

$$
\begin{aligned}
\mathbb{P}\left(v_{i} \notin \mathcal{B}_{x_{0}}\left(\mathrm{r}_{n}\right) \mid x_{i}, r_{v_{i}, x_{i}}=d_{\ell}\right) & =\mathbb{P}\left(r_{v_{i}, x_{0}}>\mathrm{r}_{n} \mid x_{i}, r_{v_{i}, x_{i}}=d_{\ell}\right) \\
& =1-\frac{1}{\pi} \arccos \left(\frac{r_{x_{i}, x_{0}}^{2}+d_{\ell}^{2}-r_{n}^{2}}{2 r_{x_{i}, x_{0}} d_{\ell}}\right)
\end{aligned}
$$

where (56) follows from invoking $r_{v_{i}, x_{0}}$ and then evaluating the probability by making use of the uniform distribution of $\beta$ in $[0, \pi]$. Finally, for $r_{x_{i}, x_{0}}>\mathbf{r}_{n}+d_{\ell}, \mathbb{P}\left(v_{i} \notin \mathcal{B}_{x_{0}}\left(r_{n}\right) \mid x_{i}, r_{v_{i}, x_{i}}=d_{\ell}\right)=1$. 
Putting (47) and (53) into (46), we get

$$
\begin{aligned}
\mathbb{P}(\hat{\Phi} & \left.\in V \mid x_{0} \in \Phi, r_{v_{0}, x_{0}}=d_{n}\right) \\
& =\exp \left(-\lambda \pi \mathrm{r}_{n}^{2}-\lambda \sum_{\ell=1}^{N} p_{\ell} \int\left(1-\mathbb{P}\left(v_{i} \notin \mathcal{B}_{x_{0}}\left(r_{n}\right) \mid x_{i}, r_{v_{i}, x_{i}}=d_{\ell}\right)\right) \mathrm{d} x_{i}\right)
\end{aligned}
$$

with integration over $\mathcal{B}_{x_{0}}\left(r_{n}+d_{\ell}\right) \backslash \mathcal{B}_{v_{0}}\left(r_{n}\right)$ and then, plugging (57) into (44), we arrive at (15), the co-channel link density of ITLinQ type I.

We now derive $\lambda_{\Psi_{\mathrm{II}}}$. Conditioned on mark $m$, the transmitters (or links) with priority lower than $m$ can be viewed as an independently sampled version of the process $\Phi$ and the density of links with a lower mark than $m$ is $m \lambda$. By leveraging the derivation of type I density (cf. (15)), the probability of retaining a given link with priority $m$ is $e^{-m \lambda A\left(\mathrm{r}_{n}, d_{\ell}\right)}$ with $A\left(\mathrm{r}_{n}, d_{\ell}\right)$ given in (17). Unconditioning on $m$,

$$
\lambda_{\Psi_{\mathrm{II}}}=\lambda \sum_{n=1}^{N} \int_{0}^{1} e^{-m \lambda A\left(\mathrm{r}_{n}, d_{\ell}\right)} \mathrm{d} m
$$

which yields (16), the co-channel link density of ITLinQ type II.

\section{APPENDIX B}

\section{Proof of Proposition 1}

The link spectral efficiency averaged over all geometries is computed as

$$
\bar{C}(\lambda, M, \mu)=\mathbb{E}\left[C\left(\rho_{0}\right)\right]
$$

where the expectation is over $\rho_{0}$. Expanding the above equation

$$
\begin{aligned}
\bar{C}(\lambda, M, \mu) & =\mathbb{E}\left[\mathbb{E}\left[\log _{2}\left(1+\operatorname{SINR}_{0} \mid \rho_{0}\right)\right]\right] \\
& =\mathbb{E}\left[\int_{0}^{\infty} \mathbb{P}\left[\log _{2}\left(1+\operatorname{SINR}_{0} \mid \rho_{0}\right)>t\right] \mathrm{d} t\right] \\
& =\mathbb{E}\left[\int_{0}^{\infty} \frac{\log _{2} e}{\gamma+1}\left(1-F_{\operatorname{SINR}_{0} \mid \rho_{0}}(\gamma)\right) \mathrm{d} \gamma\right] \\
& =\sum_{n=1}^{N} q_{n} \int_{0}^{\infty} \frac{\log _{2} e}{\gamma+1}\left(1-F_{\mathrm{SINR}_{0} \mid r_{0}, x_{0}}=d_{n}(\gamma)\right) \mathrm{d} \gamma
\end{aligned}
$$

where the outer and inner expectations in (60) are over $\rho_{0}$ and over the fading, respectively, while (62) follows from the variable change $t=\log _{2}(1+\gamma)$ and $q_{n}$ is the fraction of co-channel links with distance $d_{n}$. 
To compute the average link spectral efficiency, it is more convenient to replace the average interference $\overline{\sigma_{0, \text { out }}^{2}}$ with its exact value $\sigma_{0 \text {,out }}^{2}$ in the definition of $\rho_{0}$, i.e., we don't apply the averaging over $K$ dominant interferers. This relaxation can only make the model, whose goodness was already validated, even tighter. Then,

$$
\rho_{0}=\frac{P r_{v_{0}, x_{0}}^{-\eta}}{\sum_{k=1}^{\infty} P e_{k} r_{v_{0}, x_{k}}^{-\eta}+F_{\mathrm{N}} N_{0} B} .
$$

The conditional CDF of SINR , given $\left\{r_{v_{0}, x_{k}}\right\}_{k=1}^{\infty}$ and $\left\{e_{k}\right\}_{k=1}^{\infty}$, is

$$
\begin{aligned}
F_{\mathrm{SINR}_{0} \mid \rho_{0}}(\gamma) & =1-\exp \left[-\gamma\left(\frac{F_{\mathrm{N}} N_{0} B}{P r_{v_{0}, x_{0}}^{-\eta}}+\sum_{k=1}^{\infty} e_{k} r_{v_{0}, x_{k}}^{-\eta} r_{v_{0}, x_{0}}^{\eta}\right)\right] \\
& =1-\exp \left(-\frac{\gamma}{r_{v_{0}, x_{0}}^{-\eta}} \frac{F_{\mathrm{N}} N_{0} B}{P}\right) \prod_{k=1}^{\infty} \exp \left(-\gamma e_{k} r_{v_{0}, x_{k}}^{-\eta} r_{v_{0}, x_{0}}^{\eta}\right) .
\end{aligned}
$$

First, we average $F_{\mathrm{SINR}_{0} \mid \rho_{0}}(\gamma)$ over the interference, conditioned on $r_{v_{0}, x_{0}}=d_{n}$, to obtain

$$
F_{\mathrm{SINR}_{0} \mid r_{v_{0}, x_{0}}=d_{n}}(\gamma)=1-\exp \left(-\frac{\gamma}{d_{n}^{-\eta}} \frac{F_{\mathrm{N}} N_{0} B}{P}\right) \mathbb{E}\left[\prod_{k=1}^{\infty} \exp \left(-\gamma e_{k} r_{v_{0}, x_{k}}^{-\eta} d_{n}^{\eta}\right)\right]
$$

where the expectation is over the process of co-channel transmitter locations $\Psi$ of ITLinQ with density $\lambda_{\Psi}$. To proceed with the analysis, we recall Assumption 1 (cf. Section III-D) that the locations of co-channel transmitters outside the receiver's exclusion region belong to a homogeneous PPP with the density $\lambda_{\Psi}$. At this point, we can apply the PGFL of the PPP [36] to expect over the locations of all those transmitters that lie outside $\mathcal{B}_{v_{0}}\left(r_{n}\right)$ and simplify (67) into

$$
\begin{aligned}
F_{\mathrm{SINR}_{0} \mid r_{v_{0}, x_{0}}=d_{n}}(\gamma) & =1-\exp \left(-\frac{\gamma}{d_{n}^{-\eta}} \frac{F_{\mathrm{N}} N_{0} B}{P}\right) \exp \left(-2 \pi \lambda_{\Psi} \int_{\mathrm{r}_{n}}^{\infty}\left(1-e^{-\gamma d_{n}^{\eta} r^{-\eta}}\right) r \mathrm{~d} r\right) \\
& =1-\exp \left[-\frac{\gamma}{d_{n}^{-\eta}} \frac{F_{\mathrm{N}} N_{0} B}{P}+\pi \lambda_{\Psi} \mathrm{r}_{n}^{2}+\frac{2}{\eta} \pi \lambda_{\Psi} \gamma^{\frac{2}{\eta}} d_{n}^{2} \bar{\Gamma}\left(-\frac{2}{\eta}, \gamma\left(\frac{d_{n}}{\mathrm{r}_{n}}\right)^{\eta}\right)\right]
\end{aligned}
$$

where (69) follows from the variable change $\gamma r_{v_{0}, x_{0}}^{\eta} r^{-\eta}=u$. Next, plugging the resulting conditional distribution $F_{\mathrm{SINR}_{0} \mid r_{v_{0}, x_{0}}=d_{n}}(\gamma)$ into (63), we obtain the final expression for $\bar{C}(\lambda, M, \mu)$ in (28).

\section{REFERENCES}

[1] Y.-D. Lin and Y.-C. Hsu, "Multihop cellular: A new architecture for wireless communications," in Proc. IEEE INFOCOM, Mar. 2000, vol. 3, pp. 1273-1282.

[2] K. Doppler, M. Rinne, C. Wijting, C. B. Ribeiro, and K. Hugl, "Device-to-Device communication as an underlay to LTE-advanced networks," IEEE Commun. Mag., vol. 47, no. 12, pp. 42-49, Dec. 2009. 
[3] G. Fodor, E. Dahlman, G. Mildh, S. Parkvall, N. Reider, G. Miklós, and Z. Turányi, "Design aspects of network assisted Device-to-Device communications," IEEE Commun. Mag., vol. 50, no. 3, pp. 170-177, Mar. 2012.

[4] L. Lei, Z. Zhong, C. Lin, and X. Shen, "Operator controlled Device-to-Device communications in LTE-advanced networks," IEEE Trans. Wireless Commun., vol. 19, no. 3, pp. 96-104, June 2012.

[5] 3GPP TR 22.803 V1.0.0, "Feasibility Study for Proximity Services (ProSe) (Release 12)," Tech. Rep., 3rd Generation Partnership Project 3GPP, www.3gpp.org, Aug. 2012.

[6] F. Boccardi, R. W. Heath Jr., A. Lozano, T. Marzetta, and P. Popovski, "Five disruptive technology directions for 5G," IEEE Commun. Mag., vol. 52, no. 2, pp. 74-80, Feb. 2014.

[7] J. G. Andrews, S. Buzzi, W. Choi, S. Hanly, A. Lozano, A. C. K. Soong, and J. C. Zhang, "What will 5G be?," IEEE J. Select. Areas Commun., vol. 32, no. 6, pp. 1065-1082, June 2014.

[8] J. Seppälä, T. Koskela, T. Chen, and S. Hakola, "Network controlled Device-to-Device (D2D) and cluster multicast concept for LTE and LTE-A networks," in Proc. IEEE Wireless Commun. and Networking Conf., Mar. 2011, pp. 986-991.

[9] X. Lin, R. Ratasuk, A. Ghosh, and J. G. Andrews, "Modeling, analysis, and optimization of multicast Device-to-Device transmissions," IEEE Trans. Wireless Commun., vol. 13, no. 8, pp. 4346-4359, Aug. 2014.

[10] N. Golrezaei, A. Molisch, A. G. Dimakis, and G. Caire, "Femtocaching and Device-to-Device collaboration: A new architecture for wireless video distribution,” IEEE Commun. Mag., vol. 51, no. 4, pp. 142-149, Apr. 2013.

[11] N. Golrezaei, P. Mansourifard, A. Molisch, and A. G. Dimakis, "Base-station assisted Device-to-Device communications for high-throughput wireless video networks," IEEE Trans. Wireless Commun., vol. 13, no. 7, pp. 3665-3676, July 2014.

[12] X. Bao, U. Lee, I. Rimac, and R. R. Choudhury, "Data Spotting: Offloading cellular traffic via managed Device-to-Device data transfer at data spots," ACM SIGMOBILE Mobile Comput. Commun. Rev., vol. 14, no. 3, pp. 37-39, July 2010.

[13] N. K. Pratas and P. Popovski, "Underlay of low-rate machine-type D2D links on downlink cellular links," in Proc. IEEE ICC Workshop on M2M Communications for Next Generation IoT, June 2014, pp. 423-428.

[14] C.-W. Hsu and H.-Y. Hsieh, "Design and analysis for effective proximal discovery in Machine-to-Machine wireless networks," in Proc. IEEE ICC Workshop on M2M Communications for Next Generation IoT, June 2014, pp. 477-482.

[15] M. S. Corson, R. Laroia, J. Li, T. Richardson, and G. Tsirtsis, “Toward proximity-aware internetworking," IEEE Wireless Commun. Mag., vol. 17, no. 6, pp. 26-33, Dec. 2010.

[16] M. S. Corson, R. Laroia, J. Li, V. D. Park, T. Richardson, G. Tsirtsis, and S. Uppala, "Flashlinq: Enabling a mobile proximal internet," IEEE Wireless Commun. Mag., vol. 20, no. 5, pp. 110-117, Oct. 2013.

[17] D. Feng, L. Lu, Y. Yuan-Wu, G.Y. Li, G. Feng, and S. Li, "Device-to-Device communications underlaying cellular networks," IEEE Trans. Commun., vol. 61, no. 8, pp. 3541-3551, Aug. 2013.

[18] S. Shalmashi, G. Miao, and S. B. Slimane, "Interference management for multiple Device-to-Device communications underlaying cellular networks,” in Proc. IEEE Int. Symp. Pers., Indoor, Mobile Radio Commun., Sept. 2013, pp. $223-227$.

[19] B. Kaufman and B. Aazhang, "Cellular networks with an overlaid device to device network," in Proc. Annual Asilomar Conf. Signals, Syst., Comp., Oct. 2008.

[20] D. Wu, J. Wang, R. Q. Hu, Y. Cai, and L. Zhou, "Energy-efficient resource sharing for mobile Device-to-Device multimedia communications," IEEE Trans. Veh. Technol., vol. 63, no. 5, pp. 2093-2103, June 2014.

[21] G. George, R. K. Mungara, and A. Lozano, "An analytical framework for Device-to-Device communication in cellular networks," Available online: http://arxiv.org/abs/1407.2201/, 2014.

[22] A. Hasan and J. Andrews, "The guard zone in wireless ad hoc networks," IEEE Trans. Wireless Commun., vol. 2, pp. 897-906, Mar. 2007.

[23] F. Baccelli, J. Li, T. Richardson, S. Shakkottai, S. Subramanian, and X. Wu, "On optimizing CSMA for wide area ad-hoc networks," in Proc. Int. Symp. on Modelling and Opt. in Mobile, Ad-hoc and Wireless Networks, May 2011, pp. 354-359. 
[24] D. Torrieri and M. C. Valenti, "Exclusion and guard zones in DS-CDMA Ad Hoc networks," IEEE Trans. Commun., vol. 61, no. 6, pp. 2468-2476, June 2013.

[25] G. Alfano, M. Garetto, and E. Leonardi, "New directions into the stochastic geometry analysis of dense CSMA networks," IEEE Trans. Mobile Comput., vol. 13, no. 2, pp. 324-336, Feb. 2014.

[26] M. Haenggi, "Mean interference in hard-core wireless networks," IEEE Commun. Lett., vol. 15, no. 8, pp. 792-794, Aug. 2011.

[27] Y. Zhong, W. Zhang, and M. Haenggi, "Stochastic analysis of the mean interference for the RTS/CTS mechanism," in Proc. IEEE Int. Conf. Commun., June 2014, pp. 1996-2001.

[28] C. H. Lee and M. Haenggi, "Interference and outage in Poisson cognitive networks," IEEE Trans. Wireless Commun., vol. 11, no. 4, pp. 1392-1401, Apr. 2012.

[29] S. Cho and W. Choi, "Relay cooperation with guard zone to combat interference from an underlaid network," in Proc. IEEE Global Commun. Conf., Dec. 2011, pp. 1-5.

[30] X. Wu, S. Tavildar, S. Shakkottai, T. Richardson, J. Li, R. Laroia, and A. Jovicic, "FlashLinQ: A synchronous distributed scheduler for peer-to-peer ad hoc networks," in Proc. Annual Allerton Conf. Commun., Cont., Computing, Sept. 2010, pp. $514-521$.

[31] X. Wu, S. Tavildar, S. Shakkottai, T. Richardson, J. Li, R. Laroia, and A. Jovicic, "FlashLinQ: A synchronous distributed scheduler for peer-to-peer ad hoc networks," IEEE/ACM Trans. Networking, vol. 21, no. 4, pp. 1215-1228, Aug. 2013.

[32] N. Naderializadeh and A. S. Avestimehr, "ITLinQ: A new approach for spectrum sharing in Device-to-Device communication systems," IEEE J. Select. Areas Commun., vol. 32, no. 6, pp. 1139-1151, June 2014.

[33] R. K. Mungara, X. Zhang, A. Lozano, and R. W. Heath Jr., "On the spatial spectral efficiency of ITLinQ," in Proc. Annual Asilomar Conf. Signals, Syst., Comp., Nov. 2014.

[34] R. K. Mungara, X. Zhang, A. Lozano, and R. W. Heath Jr., "Performance evaluation of ITLinQ and FlashLinQ for overlaid device-to-device communication," in Proc. IEEE ICC Workshop on Device-to-Device Communication for Cellular and Wireless Networks (ICC'15), June 2015.

[35] F. Baccelli, B. Blaszczyszyn, and P. Muhlethaler, "Stochastic analysis of spatial and opportunistic Aloha," IEEE J. Select. Areas Commun., vol. 27, no. 7, pp. 1105-1119, Sept. 2009.

[36] M. Haenggi, Stochastic Geometry for Wireless Networks, Cambridge Univ. Press, Cambridge, U. K., 2012.

[37] M. Haenggi, "Local delay in Poisson networks with and without interference," in Proc. Annual Allerton Conf. Commun., Cont., Computing, Sept. 2010, pp. 1482-1487.

[38] F. Baccelli, J. Li, T. Richardson, S. Shakkottai, S. Subramanian, and X. Wu, "On optimizing CSMA for wide area ad-hoc networks," in Proc. Int. Symp. on Modelling and Opt. in Mobile, Ad-hoc and Wireless Networks, May 2011, pp. 354-359.

[39] X. Lin, J. G. Andrews, and A. Ghosh, "Spectrum sharing for Device-to-Device communication in cellular networks," IEEE Trans. Wireless Commun., vol. 13, no. 12, pp. 6727-6740, Dec. 2014.

[40] N. Lee, F. Baccelli, and R. W. Heath Jr., "Spectral efficiency scaling laws in dense random wireless networks with multiple receive antennas," [Online]. Available: http://arxiv.org/abs/1410.7502, 2014.

[41] P. Madhusudhanan, J. G. Restrepo, Y. E. Liu, and T. X. Brown, "Carrier to Interference ratio analysis for the shotgun cellular system,” in Proc. IEEE Global Commun. Conf., Honolulu, USA, Nov. 2009, pp. 1-6.

[42] R. K. Mungara, D. Morales-Jiménez, and A. Lozano, "System-level performance of interference alignment," IEEE Trans. Wireless Commun., vol. 14, no. 2, pp. 1060-1070, Feb. 2015.

[43] G. George, R. K. Mungara, and A. Lozano, "Overlaid Device-to-Device communication in cellular networks," in IEEE Global Commun. Conf. (GLOBECOM'14), Dec. 2014. 
[44] A. Lapidoth and S. Shamai, "Fading channels: how perfect need "perfect side information" be?," IEEE Trans. Inform. Theory, vol. 48, no. 5, pp. 1118-1134, May 2002.

[45] I. Shomorony and A. S. Avestimehr, "Worst-case additive noise in wireless networks," IEEE Trans. Inform. Theory, vol. 59, no. 6, pp. 3833-3847, June 2013.

[46] M. Haenggi, "On distances in uniformly random networks," IEEE Trans. Inform. Theory, vol. 51, no. 10, pp. 3584-3586, Oct. 2005.

[47] A. Lozano and N. Jindal, "Are yesterday's information-theoretic fading models and performance metrics adequate for the analysis of today's wireless systems?," IEEE Commun. Mag., vol. 50, no. 11, pp. 210-217, Nov. 2012.

[48] H. Yoon, J. S. Kim, S. J. Bae, B. Choi, and M. Y. Chung, "Performance analysis of FlashLinQ with various yielding threshold values," in Proc. Int. Conf. ICT Convergence, Oct. 2012, pp. 477-478.

[49] X. Zhang and J. G. Andrews, "Downlink cellular network analysis with multi-slope path loss models," [Online]. Available: http://arxiv.org/abs/1408.0549, 2014.

[50] D. Stoyan, W. S. Kendall, and J. Mecke, Stochastic Geometry and its Applications, John Wiley \& Sons Ltd, New York, USA, 1995. 